\title{
Influence of grid resolution on the spectral characteristics of noise radiated from turbulent jets: sound pressure fields and their decomposition
}

\author{
M. Angelino ${ }^{\mathrm{a}, *}$, H. Xia ${ }^{\mathrm{a}}$, G. J. Page ${ }^{\mathrm{a}}$ \\ ${ }^{a}$ Department of Aeronautical and Automotive Engineering, Loughborough University, United Kingdom
}

\begin{abstract}
Jet noise remains a key target for aircraft noise reduction in the foreseeable future. While being extremely challenging, the requirement of predicting both low and high frequency noise spectra is increasingly important for design purposes. Novel approaches are needed to overcome the current numerical limitations in capturing the required broad noise spectra. Once sufficiently resolved, the energy contents of the numerically simulated near- and far-field sound pressure have intrinsic correlations among different levels of grid resolution. The present work explores the potential of such correlations to broaden the spectral prediction. The noise radiated from high subsonic turbulent jets is investigated using large-eddy simulation. The 3-D filtered compressible Navier-Stokes solutions are obtained for an axisymmetric and a serrated nozzle on successively refined multi-resolution grids, ranging from 5 to 80 million grid points. The radiated far-field sound is computed using the Ffowcs Williams - Hawkings (FW-H) surface integral method. Fourier decomposition for pressure near-field is applied to help identify the location of the sound source regions and the dominant directions of propagation, which provides a more thorough understanding of the effect of the grid resolution on the numerical cut-off frequencies of the far-field spectra. Further analysis of the far-field spectra and of their azimuthal modes confirms that a novel strategy to obtain a broadened overall sound spectrum is possible, at reduced computational cost, from a combination of multiple spectra from successively refined grids.
\end{abstract}

Keywords: Jet noise, Turbulence, Large-Eddy Simulation (LES), Ffowcs Williams - Hawkings (FW-H) method

\section{Introduction}

The future growth of aviation is closely connected to aircraft environmental impact. Noise reduction is one of the industry's main concerns due to goals such as the $65 \%$ reduction of noise emissions by the

\footnotetext{
* Corresponding author

Email address: m.angelino@lboro.ac.uk (M. Angelino)

Preprint submitted to Computers \& Fluids
} 


\begin{tabular}{|llll|}
\hline \multicolumn{2}{|l|}{ Nomenclature } & & \\
$a_{\infty}$ & speed of sound & St & Strouhal number \\
$C_{m}$ & azimuthal Fourier coefficients & $T_{\infty}$ & ambient temperature \\
$D_{j}$ & jet diameter & $t^{*}$ & reference time \\
$E_{11}$ & streamwise energy spectrum & $T_{j}$ & jet exit temperature \\
$\mathbf{F}$ & inviscid flux tensor & $U$ & axial velocity \\
$f$ & frequency & $U_{j}$ & jet exit velocity \\
$m$ & azimuthal mode & $u^{\prime}$ & axial velocity fluctuation \\
Ma & Mach number & $\overline{u^{\prime} u^{\prime}}$ & axial normal stress \\
$n_{\theta}$ & number of azimuthal points & $x, y, z$ & Cartesian coordinates \\
OASPL & overall sound pressure level & $r_{0.5}$ & jet half-width \\
$p \prime$ & acoustic pressure fluctuation & $\delta^{*}$ & displacement thickness \\
$p_{\infty}$ & ambient pressure & $\varepsilon$ & upwinding parameter \\
PSD & power spectral density & $\theta$ & polar angle \\
$\mathbf{Q}$ & primary flow variable vector & $\nu_{s g s}$ & subgrid-scale viscosity \\
$r$ & radial distance & $\sigma$ & singular value of velocity gradient tensor \\
$R_{11}$ & auto-correlation function of $u^{\prime}$ & $\phi$ & azimuthal angle \\
Re & Reynolds number & & \\
& &
\end{tabular}

year 2050 set by the European Commission [1]. For a civil turbofan transport aircraft, the jet exhaust is a significant component of the overall aircraft noise - particularly at take-off conditions. The understanding of jet noise, and hence the ability to predict accurately the noise from future designs, is crucial. In particular, computational methods have the potential to replace expensive testing of models in anechoic facilities, and, just as importantly, to provide additional insight into the sound generation and propagation processes. This work presents a spectral analysis of near- and far-field noise and proposes an approach to combine numerical simulations with different temporal and spatial resolutions to increase the frequency range with only a small increase in computational cost.

Since the pioneering work of Lighthill $[2,3]$, numerous analytical and experimental studies [4] have attempted to provide a more solid understanding of the jet noise generation mechanism. Recent advances in computational aeroacoustics [5] have allowed researchers to tackle the complexity of the phenomenon numerically under controlled conditions. Large-eddy simulation (LES) has proven to be a robust tool for prediction of the jet acoustic field [6-11]. Relying on direct computation of the far-field noise is often not computationally affordable. Integral approaches, like Kirchhoff [12] or Ffowcs Williams - Hawkings [13] 
(FW-H), allow the evaluation of the sound propagation from the perturbation field captured on a surface surrounding the jet, thus requiring an accurate solution only of the flow enclosed within the surface. The FW-H method has been validated in numerous studies [14-20] and has also been applied to jets from complex geometries, such as serrated nozzles [21, 22].

Serrated (or chevron) nozzles are of great interest to the aerospace industry. Experiments [23-28] have shown that serration modification to the round nozzle can bring as much as $6 \mathrm{~dB}$ reduction in peak noise during take-off with less than $0.5 \%$ thrust loss during cruise. Large-eddy simulations [29-33] have revealed the fundamentally different mechanisms of shear layer growth, with increased mixing caused by the nozzle geometry. Chevrons were also found to reduce the jet and wing/flap interactions [34]. Understanding how grid resolution affects the noise spectrum in jets from both round and serrated nozzles is therefore crucial, and can play an important role in the design optimization of future jet engine nozzles.

For a medium-sized engine the spectral range requirement of Strouhal number (the non-dimensional frequency, $\left.\mathrm{St}=f D_{j} / U_{j}\right)$ goes from 0.01 to $10[31,35]$. Low frequencies are particularly important when taking into account jet-flap interaction [36-39], but predicting the high-frequency noise (up to St $=8 \sim 10$ ) poses the biggest challenge for current numerical methods.

For LES, the highest achievable frequency is restricted by the grid resolution, whilst the lowest frequency is limited by the ability to capture sufficient periods leading to the computation of large numbers of time steps. Merely increasing the resolution has a large impact on the overall cost. For example, to double the highest resolvable Strouhal number will broadly mean a halving of the grid size in each spatial direction, leading to an increase in computational cost of a factor of 8 for a single time step. However, the time step will also need to be halved, so to maintain the same low frequency requirement leads to an in increase in cost of a factor of approximately 16 .

As an example of this problem, a well-resolved fine-grid solution [30, 40], which is potentially able to capture the high-frequency spectrum, is often too costly to run for a sufficiently long period of time to be statistically converged at low frequencies. The fine solution typically produces much better high-frequency predictions but performs poorly at low frequencies, whereas the coarser solution spectrum decays far too quickly at high frequencies but does provide good low-frequency predictions simply because it has been possible to run for a much longer physical time, due to reduced computational requirements.

This work is intended to provide a series of numerical observations on the near-field acoustics and the far-field FW-H results from LES solutions obtained with carefully constructed multi-resolution grids. The goal is to investigate whether there is a level of grid refinement which is sufficient to give accurate low and 

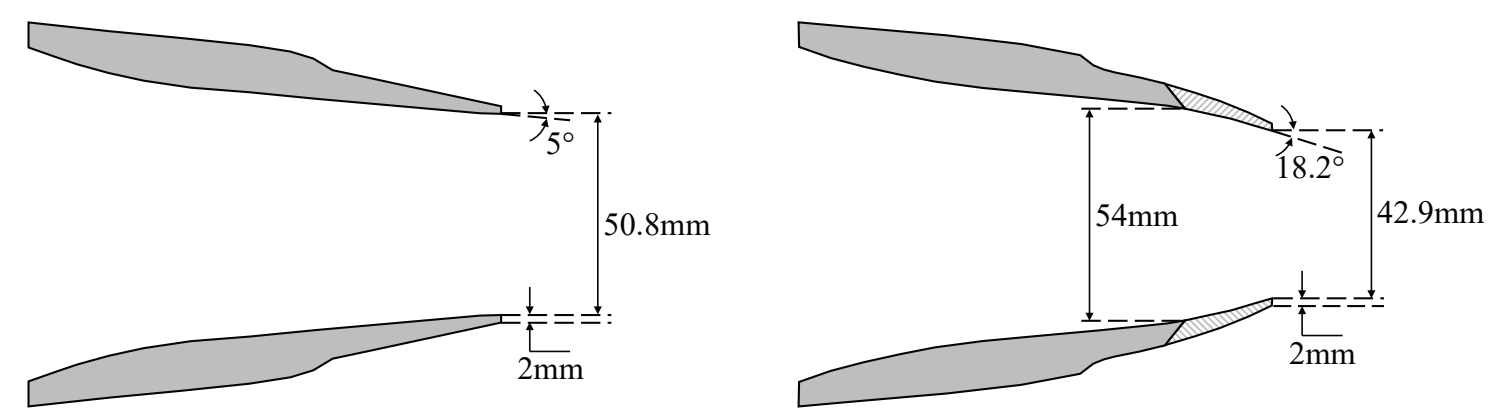

Figure 1: Geometry of NASA Glenn nozzles SMC000 on the left and SMC006 on the right (chevron notch profile with solid fill, tip profile with striped pattern).

medium frequency results, and to what extent further refinement leads to improved high frequency results, with the intention of laying the foundations for a strategy to increase the overall accuracy by combining low-frequency results from coarser grids with high-frequency results from finer grids.

The paper is structured as follows. Section 2 defines the jet configuration, the meshing strategy and the numerical methods. Simulation results for the turbulent flow fields from round and chevron nozzles are discussed in Sections 3. Section 4 presents the acoustic analysis of near-field pressure and far-field noise propagation, and a discussion on the strategy to improve the range of resolvable frequencies. Finally, concluding remarks are provided in Section 5.

\section{Simulation method and parameters}

\subsection{Jet configuration and meshing strategy}

The present study focuses on a cold jet discharging into stagnant ambient flow conditions (setpoint 7 of Tanna [41]). These conditions are widely used in jet noise studies, with an acoustic Mach number at the jet exit $\mathrm{Ma}=U_{j} / a_{\infty}=0.9$ and a static temperature ratio $T_{j} / T_{\infty}=0.84$. The ambient conditions are $p_{\infty}=0.97 \times 10^{5} \mathrm{~Pa}$ and $T_{\infty}=280.2 \mathrm{~K}$. The Reynolds number is approximately $10^{6}$ based on the nozzle exit diameter $D_{j}$ and jet exit velocity $U_{j}$. The axisymmetric nozzle SMC000 has a $50.8 \mathrm{~mm}$ exit diameter while the serrated SMC006 has a slightly reduced effective jet diameter due to the inward bending of the chevrons (see Figure 1). SMC006 is serrated in the circumferential direction with six equally spaced chevron tips and six notches between each chevron corresponding to a $\pi / 3$ sector, and is placed in a position so that planes $z=0$ and $y=0$ cut through a pair of tips and notches, respectively.

Solutions are obtained on grids with hexahedral elements, ranging from 5 to 10, 20, 40 and 80 million grid points. Mesh refinement follows an equal-ratio rule, i.e. the number of cells increases every time by a factor 

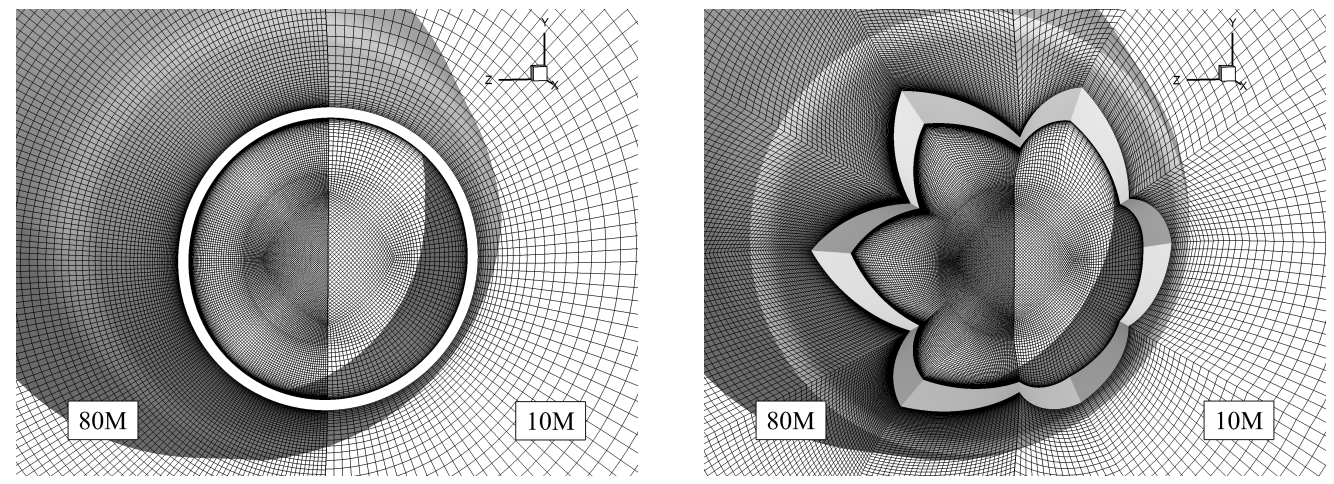

Figure 2: Detail of a $y$ - $z$ cut-plane mesh at nozzle exit for the SMC000 nozzle on the left, and the SMC006 nozzle on the right. The left half of each image is from the $80 \mathrm{M}$ grid, the right half from the $10 \mathrm{M}$.
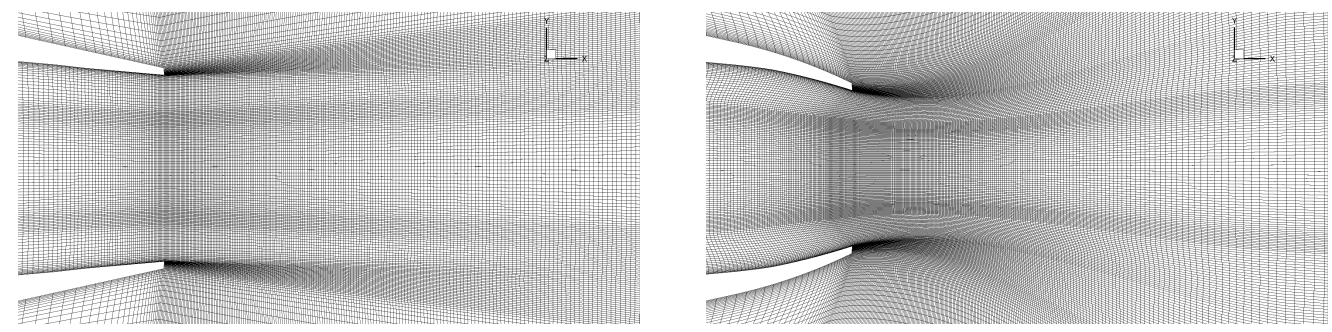

Figure 3: Detail of a $x-y$ cut-plane mesh at nozzle exit for cases R0-10M (left) and S6-10M (right).

of $\sqrt[3]{2}$ along all three directions. Hence, for example, the $80 \mathrm{M}$ grid is exactly twice as fine as the $10 \mathrm{M}$ grid in every direction (see Figure 2). Particular attention has been paid to the clustering of cells along the edges of the grid blocks. The first and last cell spacings are refined by the same factor $\sqrt[3]{2}$, while a smooth evolution of spacing and growth rate inside the domain (see Figure 3) is ensured by the point distribution described in Appendix A. Table 1 defines the case IDs and reports relevant grid parameters for all simulations.

The computational domain consists of upstream, jet inlet, far-field, and downstream boundaries. The domain is $72 D_{j}$ long axially, with a radial extent of $25 D_{j}$ at the upstream boundary and $50 D_{j}$ at the downstream end (see Figure 4). On the nozzle solid wall, no-slip and adiabatic thermal conditions are applied. The LES domain comprises non-reflective BCs in the far-field and "sponge" zones with ramped numerical dissipation towards the downstream boundary.

The time step was set to one thousandth of the reference time $t^{*}=D_{j} / U_{j}$, to ensure accuracy up to a maximum theoretical Strouhal number of 500 according to the Nyquist theorem. Stability was guaranteed by an implicit time scheme (as will be described in more detail in Section 2.2). The time step refinement follows the same refinement ratio as the cell size, so that it decreases by $\sqrt[3]{2}$ times from one grid to the next. After $100-200 t^{*}$ to initialize the jet flow, a further $100-300 t^{*}$ is needed to obtain converged evaluations of turbulent 


\begin{tabular}{lccccccc}
\hline Case ID & \multirow{2}{*}{ nozzle } & grid points & $n_{\theta}$ & $\begin{array}{c}\Delta x / \delta^{*} \\
\text { at } x=0\end{array}$ & $\begin{array}{c}\text { growth rate } \\
\partial \Delta x / \partial x\end{array}$ & $\begin{array}{c}\Delta r / \delta^{*} \\
\text { at } r=D_{j} / 2, x=0\end{array}$ & $\begin{array}{c}\text { growth rate } \\
\partial \Delta r / \partial x\end{array}$ \\
\hline R0-05M & SMC000 & $5,156,644$ & 120 & 0.287 & 1.01 & 0.0577 & 1.0126 \\
R0-10M & SMC000 & $10,586,144$ & 152 & 0.229 & 1.0079 & 0.0457 & 1.01 \\
R0-20M & SMC000 & $20,673,984$ & 192 & 0.181 & 1.0063 & 0.0363 & 1.0079 \\
R0-40M & SMC000 & $41,073,727$ & 240 & 0.145 & 1.005 & 0.0287 & 1.0063 \\
R0-80M & SMC000 & $84,325,770$ & 304 & 0.116 & 1.004 & 0.0229 & 1.005 \\
\hline S6-05M & SMC006 & $5,346,912$ & 156 & 0.0696 & 1.02 & 0.0139 & 1.018 \\
S6-10M & SMC006 & $10,409,304$ & 198 & 0.0552 & 1.0159 & 0.011 & 1.014 \\
S6-20M & SMC006 & $20,643,273$ & 246 & 0.0442 & 1.0126 & 0.00872 & 1.011 \\
S6-40M & SMC006 & $40,574,296$ & 312 & 0.0353 & 1.01 & 0.00696 & 1.009 \\
S6-80M & SMC006 & $80,331,332$ & 396 & 0.0276 & 1.0079 & 0.00552 & 1.007 \\
\hline
\end{tabular}

Table 1: Parameters of all numerical grids. $\delta^{*}$ is the displacement thickness of the mixing layer at $x / D_{j}=0.05(\operatorname{chevron}$ root mixing layer for the serrated case).

\begin{tabular}{lccc}
\hline Case ID & $\begin{array}{c}\text { time step } \\
\Delta t / t^{*}\end{array}$ & $\begin{array}{c}\text { integration } \\
\text { time }\left(\# t^{*}\right)\end{array}$ & $\begin{array}{c}\text { relative } \\
\text { estimated cost }\end{array}$ \\
\hline R0-05M & 0.00159 & 300 & 0.4 \\
R0-10M & 0.00126 & 300 & 1 \\
R0-20M & 0.001 & 300 & 2.52 \\
R0-40M & 0.00079 & 300 & 6.35 \\
R0-80M & 0.00063 & 50 & 2.67 \\
\hline S6-05M & 0.00159 & 300 & 0.4 \\
S6-10M & 0.00126 & 300 & 1 \\
S6-20M & 0.001 & 300 & 2.52 \\
S6-40M & 0.00079 & 300 & 6.35 \\
S6-80M & 0.00063 & 50 & 2.67 \\
\hline
\end{tabular}

Table 2: Run-time details.

statistics and the FW-H integral. The choice of total simulation time is dictated by a compromise between accuracy and computational cost. In the coarser simulations ( 5 to $40 \mathrm{M}$ cells) $300 t^{*}$ was used, yielding reliable noise estimates in the low-frequency range, as will be discussed in Section 4. The 80M case statistics were collected over a period of $50 t^{*}$ in order to obtain affordable high-frequency results. Run-time details of all cases are summarized in Table 2 .

\subsection{Numerical method}

The compressible Favre-averaged Navier-Stokes equations are solved with the code FLUXp (Flux Limited Unstructured eXtrapolation in parallel), based on a cell centred finite volume method for arbitrarily unstructured meshes. The second-order spatial discretization is based on a MUSCL approach [42] and allows for flexible and robust handling of complex geometries, while still providing adequate accuracy for LES on sufficiently refined grids.

A dual-time integral [43] is employed with the outer physical time discretized by a three-level backward Euler scheme. This leads to second-order temporal accuracy. The inner pseudo time is advanced by a 


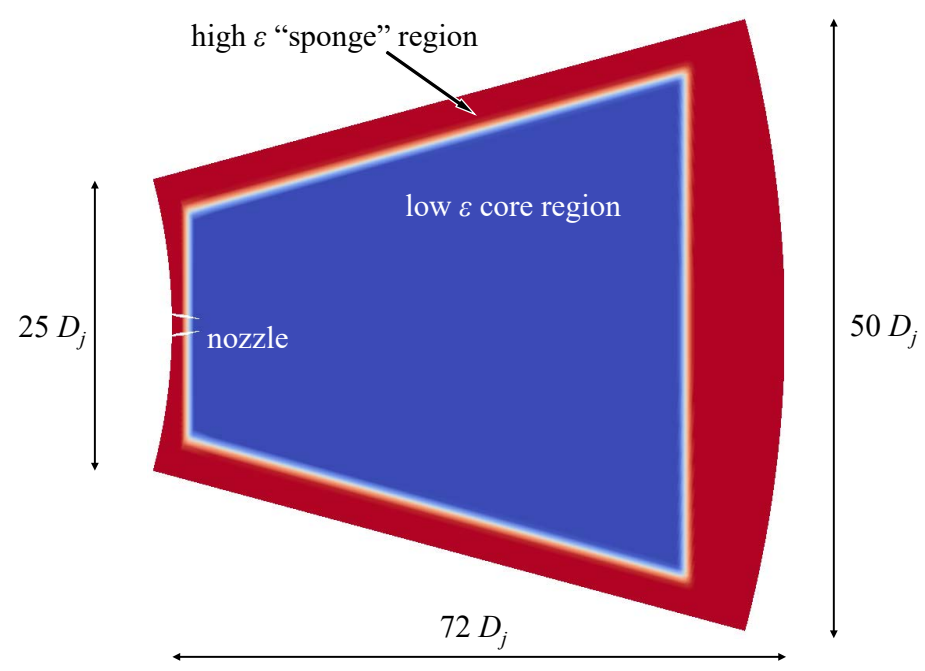

Figure 4: Domain definition and distribution of the upwinding parameter $\varepsilon$

three-stage Runge-Kutta scheme. As the outer time is discretized implicitly, larger physical time steps are allowed thus increasing the efficiency compared with explicit time marching.

While the viscous flux is discretized using a standard central difference, to compute the inviscid flux, Roe's flux difference splitting approximate Riemann solver [44] is employed at the interface between two neighbouring control volumes:

$$
\mathbf{F} \cdot \mathbf{n}=\frac{1}{2}\left(\mathbf{F}_{L}+\mathbf{F}_{R}\right) \cdot \mathbf{n}-\varepsilon \frac{1}{2}|\mathbf{A}|\left(\mathbf{Q}_{R}-\mathbf{Q}_{L}\right)
$$

In the above, $\mathbf{n}$ is the face unit normal, $|\mathbf{A}|=\mathbf{M}|\mathbf{\Lambda}| \mathbf{M}^{-1}$ is the diagonalizing transform, $\mathbf{A}=\partial(\mathbf{F} \cdot \mathbf{n}) / \partial \mathbf{Q}$ is the Jacobian, and $\mathbf{F}$ and $\mathbf{Q}$ are the inviscid flux tensor and primary flow variable vector of the filtered Navier-Stokes equations, respectively. A gradient based extrapolation is employed to compute $\mathbf{Q}_{L}$ and $\mathbf{Q}_{R}$. The inviscid flux calculation takes the form of a central difference, plus a controllable function of the primary variable vectors that acts as an upwinding term. In jet noise simulations it is crucial to reduce the level of dissipation caused by the upwinding, but without compromising stability. Different approaches to flux splitting were developed to increase robustness, especially against shocks (AUSM [45], SLAU [46], KEP [47]). In the present work, following Bui [48], $\varepsilon$ is adopted as an additional parameter to directly control the amount of upwinding (see Xia et al. [21]). Figure 4 gives the distribution of the upwinding parameter $\varepsilon$. Its maximum value of 1 corresponds to fully upwinded flux, which is used in the "sponge" zone to damp the acoustic reflections near the external boundaries. Its minimum value was set to 0.1 to avoid numerical 
instabilities. A linear transition connects the two regions with the lowest and highest $\varepsilon$ values. More details on the solver can be found in Xia [49] and Xia et al. [21].

The choice of the LES subgrid-scale (SGS) model is critical for jet applications. Standard models with constant coefficient are usually too dissipative in the initial free shear layer of the jet, thus delaying its transition. Dynamic models can overcome this issue [50-53], potentially at the price of stability, which is not always desirable when simulating complex flows. This is one reason why implicit LES (or ILES) has been widely adopted in the literature $[21,54,55]$, since the absence of excessive SGS viscosity allows accurate capture of the jet behaviour, especially near the nozzle. Alternative SGS models [56, 57] to the original Smagorinsky model can yield a more physical behaviour for boundary and free shear layers, by replacing the classical strain rate in the formulation of the subgrid-scale viscosity $\nu_{S G S}$, with other invariants of the velocity field. One of the latest examples of this type of approach is the $\sigma$-model [58], which has been validated for jet noise $[22,59]$, and is chosen for the present work. The subgrid-scale viscosity is defined as

$$
\nu_{S G S}=\left(C_{\sigma} \Delta\right)^{2} \mathcal{D}_{\sigma}
$$

where $C_{\sigma}=1.35$ is the model constant, $\Delta$ is the subgrid characteristic length scale and

$$
\mathcal{D}_{\sigma}=\frac{\sigma_{3}\left(\sigma_{1}-\sigma_{2}\right)\left(\sigma_{2}-\sigma_{3}\right)}{\sigma_{1}^{2}}
$$

where $\sigma_{1} \geq \sigma_{2} \geq \sigma_{3} \geq 0$ are the three singular values of the velocity gradient tensor. Unlike more established models of the Smagorinsky type, the $\sigma$-model has the property to automatically vanish as soon as the resolved field is two-dimensional, such as in pure shear, thus allowing for a faster development of the turbulent free shear layer of the jet, while still retaining the correct dissipation rate in fully developed turbulence further downstream. In addition, it has, by construction, the appropriate cubic behaviour in the vicinity of solid boundaries without requiring any ad-hoc treatment [58].

Recent studies focused on the effects of nozzle inflow and boundary layer turbulence on jet development and noise generation $[11,40,60,61]$. The introduction of turbulence was shown to improve the flow field and sound prediction for jets originating from a straight cylindrical pipe, at an increased computational cost and complexity due to the generation of synthetic turbulence, near-wall grid refinement and wall modelling. However, the strong sensitivity to the resulting boundary layer characteristics requires adequate knowledge of the experimental conditions, which are not available for the case studied in the present work. Additionally, the strong contraction within the SMC000 and SMC006 nozzles and the angle at the nozzle exit (see Figure 
1) generate a favourable pressure gradient which thins the nozzle boundary layer and has a stabilizing effect on the turbulence, forcing the boundary layer towards partial relaminarization [62]. The sensitivity of the jet development to the inflow conditions is therefore reduced, and its study is not considered a primary objective of this work. Therefore, in the present simulations, no boundary layer turbulence was introduced.

\subsection{Far-field acoustic computation}

For the far-field acoustic pressure fluctuation $p^{\prime}(\mathbf{x}, t)$, the FW-H surface integral [63] is computed. Since the noise source is assumed to be inside the surface (if the surface is large enough and far enough from the jet exit region), a simplification can be made by omitting the volume quadrupole integral. This, as suggested by Shur et al. [64] and Di Francescantonio [63], saves substantial data storage. The integral equation is as follows

$$
4 \pi p^{\prime}=\frac{\partial}{\partial t} \int_{S}\left[\frac{\rho u_{n}}{r}\right] d S+\frac{1}{a_{\infty}} \frac{\partial}{\partial t} \int_{S}\left[\frac{p_{n r}^{\prime}+\rho u_{n} u_{r}}{r}\right] d S+\int_{S}\left[\frac{p_{n r}^{\prime}+\rho u_{n} u_{r}}{r^{2}}\right] d S
$$

In the above, $\mathbf{r}$ ( $r$ being its modulus) defines the observer position, $a_{\infty}$ stands for the ambient speed of sound and $S$ is the FW-H surface. The quantities in the square brackets are computed at "retarded" times. Also, $n_{j}$ is the component of the unit outward normal vector on the surface, and $u_{j}$ is the velocity component.

The increased resolution of the finer grids is expected to benefit the accuracy of the far field noise in two ways: by resolving smaller scales of turbulence and reducing dissipation on the pressure fluctuations emanating from the turbulence as they travel towards the FW-H surface.

\section{Turbulent jet flow field}

This section provides a statistical description of the flow in the cases summarized in Table 2. The goal is to show, qualitatively and quantitatively, how the numerical method is consistently more accurate as grid refinement proceeds, but nonetheless yields satisfactory results on mid-range grids. This is crucial to verify that lower-frequency noise sources are well-captured even on coarser grids.

In the following sub-sections, results of the jet from the round and the serrated nozzle are reported. The data shown have been chosen in order to emphasize particular aspects of the different nozzle geometries, and at the same time offer in a concise form a complete picture of the available statistics.

\subsection{Round nozzle}

Figure 5 provides a first qualitative comparison between cases R0-10M and R0-80M. Following the refinement rule described above, from the former case to the latter the resolution is doubled along every 

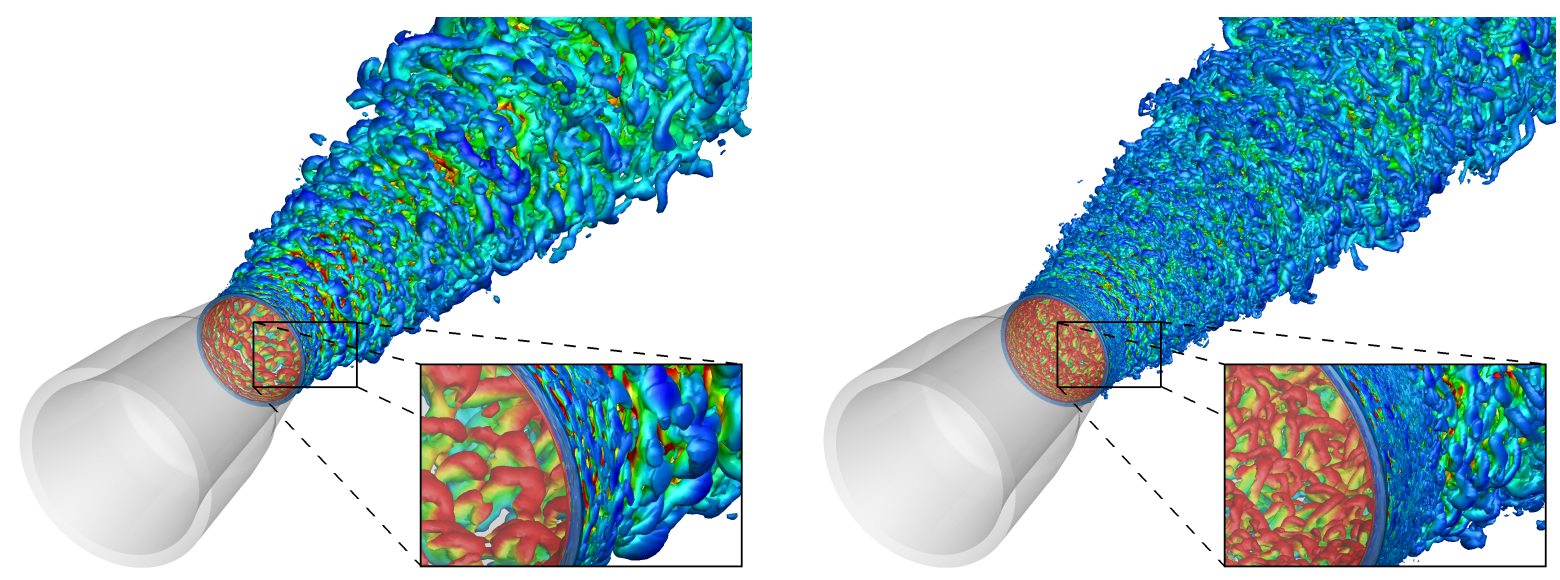

Figure 5: $Q$-criterion isosurfaces of the round case, colored by $u$. R0-10M on the left; R0-80M on the right.

direction. The vortical flow structures are depicted by isosurfaces of the $Q$-criterion [65], which defines a vortex as a region where $Q=\frac{1}{2}\left(\|\boldsymbol{\Omega}\|^{2}-\|\mathbf{S}\|^{2}\right)>0$, i.e. where the norm of the vorticity tensor $\boldsymbol{\Omega}$ dominates that of the strain-rate $\mathbf{S}$. This vortex identification method is more effective than criteria that rely only on vorticity, since these are unable to distinguish between shear rotation and swirling motion. The shear layer produced at the inner lip of the nozzle (visible in red thanks to the transparency of the nozzle geometry) breaks down into noticeably smaller structures in the $80 \mathrm{M}$ grid. There is, however, a clear similarity between the blue roller-like big structures of the two cases, which suggests that the coarser grids used in this study are able to capture the large-scale behaviour of the jet, despite the lack of smaller-scale information, as the influence of small-scale turbulence on large-scale structures is considered to be negligible [66].

Mean quantities are calculated by means of the average operator $\bar{\square}=\frac{1}{2 \pi T} \int_{0}^{2 \pi} \int_{0}^{T} \square d t d \phi$ in both time $t$ and azimuthal direction $\phi$. Figure 6 (left) compares mean axial velocity along the centerline. Symbols are measurement data from Bridges and Wernet [67]. The agreement between centerline numerical predictions and experiments is very good. The length of the potential core is slightly underpredicted on the coarser grids, whereas the $40 \mathrm{M}$ and $80 \mathrm{M}$ grids are able to properly capture the potential core length.

Figure 6 (right) shows the longitudinal evolution of the jet half-width $r_{0.5}$, defined such that $U\left(x, r_{0.5}(x)\right)=$ $0.5 U(x, 0)$. The coarser cases present an earlier jet expansion compared to the experiments, consistent with the underprediction of the potential core length, while the fine cases (40M and $80 \mathrm{M})$ are in very good agreement with the experimental results. The rate of expansion, on the other hand, is the same for all cases $\left(d r_{0.5} / d x \approx 0.066\right)$, confirming that in this region all grids are fine enough to capture the large eddies responsible for the shear layer characteristics.

The centerline and lipline normal stresses in Figure 7 show a similar improvement due to the grid 

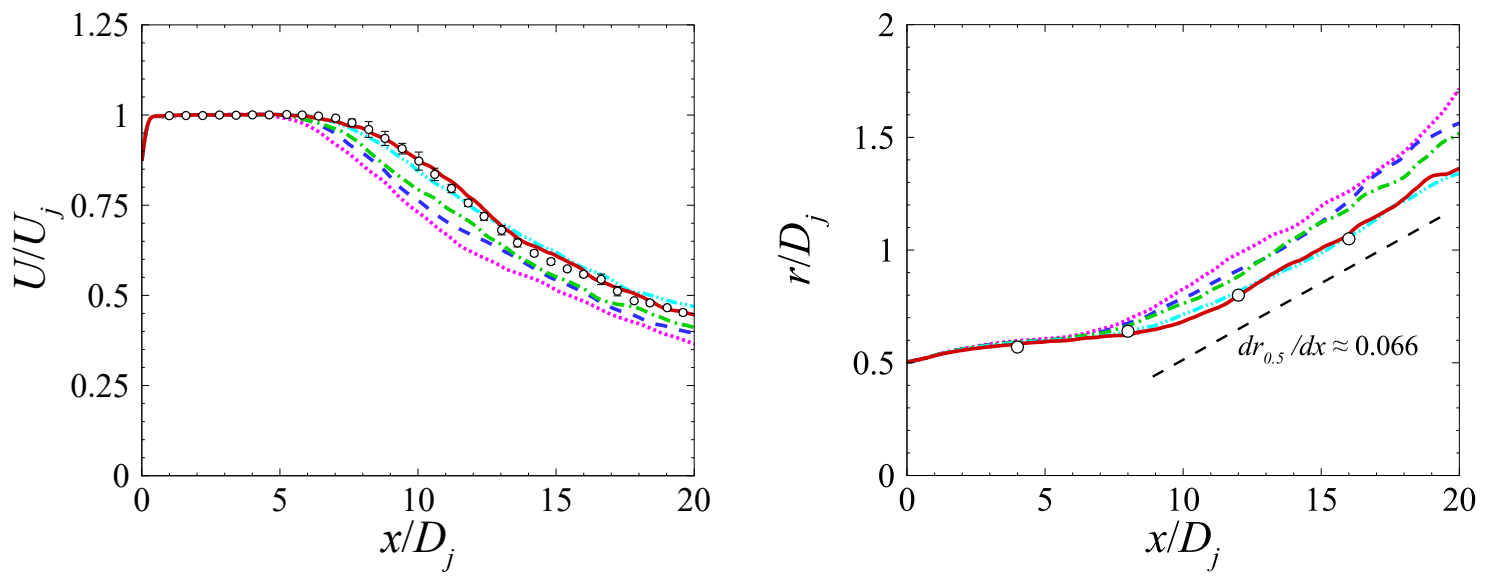

Figure 6: Centerline velocity and jet half-width for the round nozzle. Bridges and Wernet [67] $\bigcirc$, R0-05M (magenta dotted), R0-10M (blue dashed), R0-20M (green dash-dot), R0-40M (cyan dash-dot-dot), R0-80M (red solid).
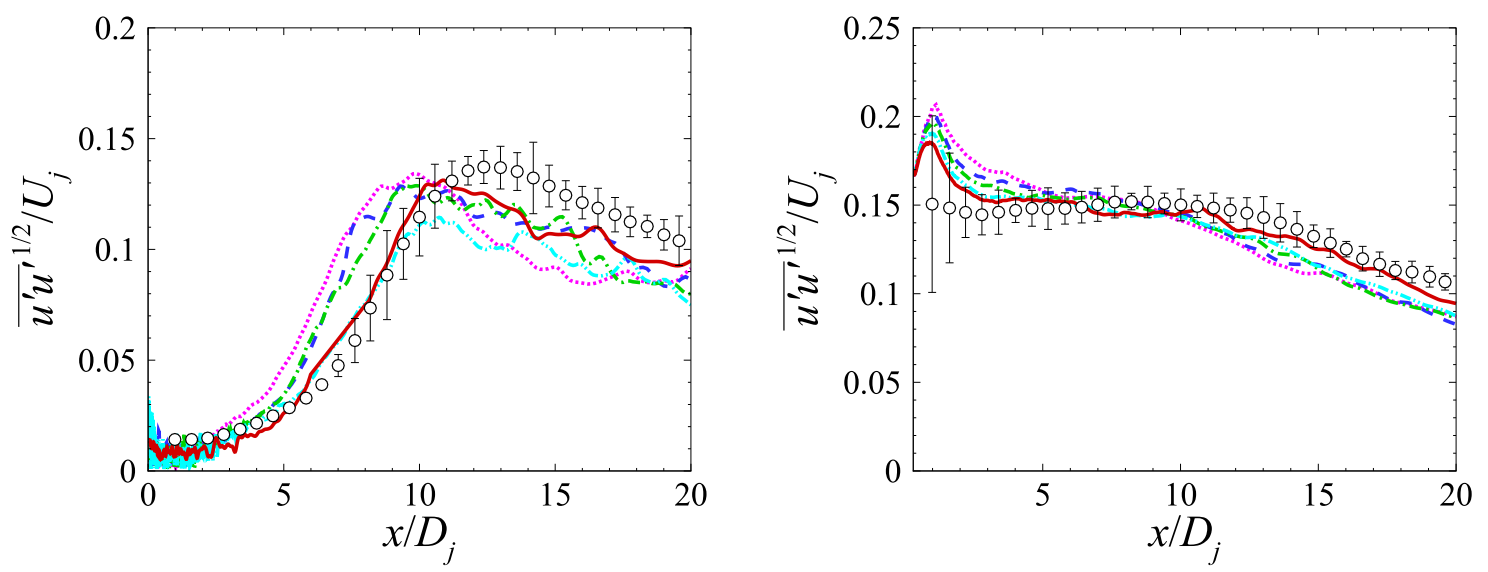

Figure 7: Centerline and lipline normal stress for the round nozzle. Bridges and Wernet [67] $\bigcirc$, R0-05M (magenta dotted), R0-10M (blue dashed), R0-20M (green dash-dot), R0-40M (cyan dash-dot-dot), R0-80M (red solid).

refinement. The fine cases $(40 \mathrm{M}$ and $80 \mathrm{M})$ accurately predict the potential core velocity fluctuations and the turbulence increase along the centerline in correspondence to the end of the potential core. The lipline normal stresses present an early overshoot typical of transitional behaviour. We believe this is due to the under-resolved turbulent fluctuations in the numerical initial shear layer in the absence of inflow turbulence. On the other hand, the nozzle contraction has the effect of partially relaminarizing the experimental boundary layer, and it is therefore unclear if a weak overshoot is to be expected. In any case, refining the grid has the beneficial effect of reducing the numerical peak normal stress, allowing for a faster transition to a turbulent shear layer. The introduction of boundary layer turbulence could produce a similar benefit, but would also entail additional complexity, and it is not the main objective of this paper. Future work is needed to quantify the effects of this limitation. 

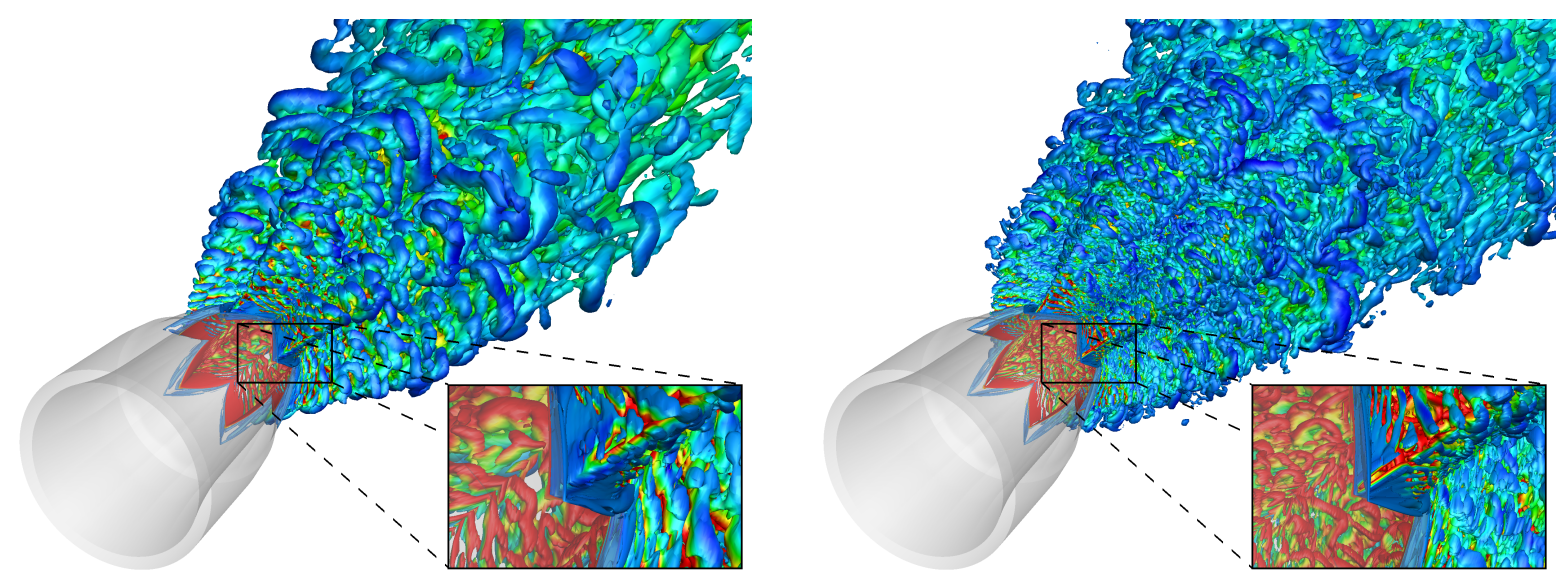

Figure 8: $Q$-criterion isosurfaces of the serrated case, colored by $u$. S6-10M on the left; S6-80M on the right.
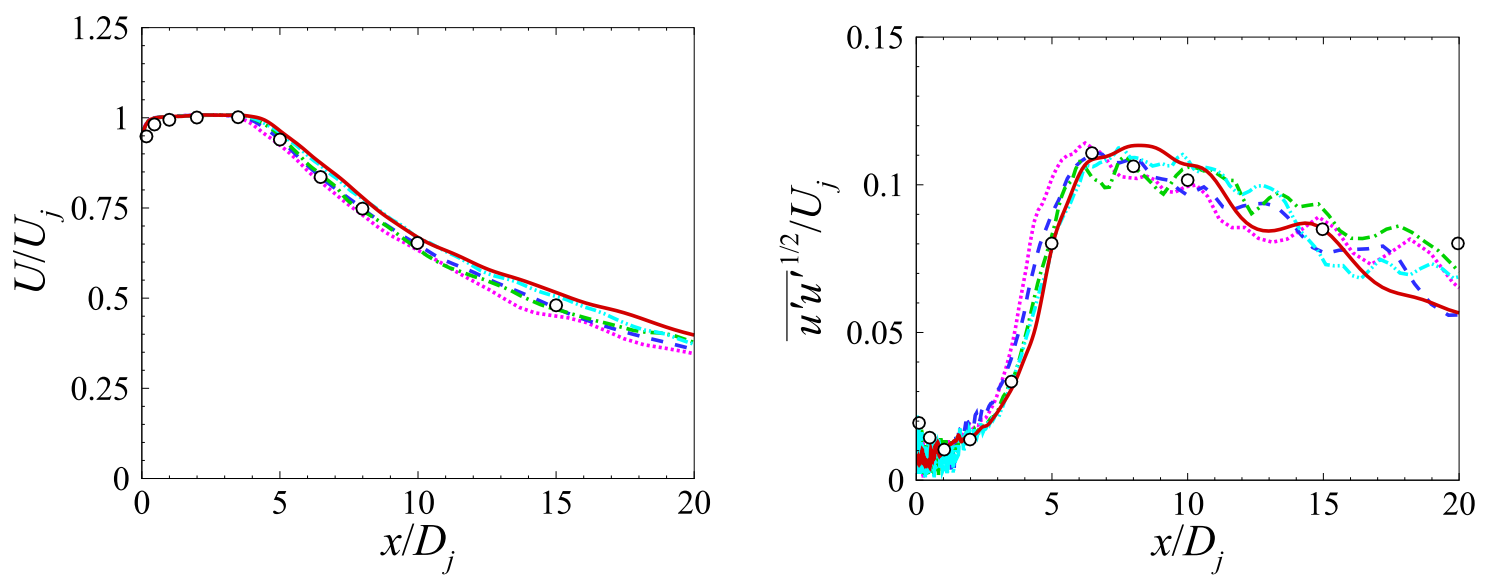

Figure 9: Centerline velocity and normal stress for the chevron nozzle. Bridges and Brown [24] $\bigcirc$, S6-05M (magenta dotted), S6-10M (blue dashed), S6-20M (green dash-dot), S6-40M (cyan dash-dot-dot), S6-80M (red solid).

\subsection{Serrated nozzle}

In the serrated cases the shear layer grows with a fundamentally different mechanism. The flow past the tips tends to go inward, whereas the flow through the notches moves outward (see Figure 8) creating extra stream-wise vorticity, hence more mixing, which is captured even in the coarser cases. The potential core is notably shortened and all grids present centerline statistics in excellent agreement with experiments, as shown in Figure 9.

Mean velocity profiles for the serrated case are plotted in Figure 10, for a cut plane through a chevron root. An average operation is performed on the six periodic azimuthal root planes. Streamwise locations are $x / D_{j}=0.5,1,2.5,5$ and 10 . Individual velocity profiles are separated by a horizontal offset of 1 . The agreement with experiments is good for all grids, with some discrepancy at $x / D_{j}=0.5,1$ and 2.5 in the 

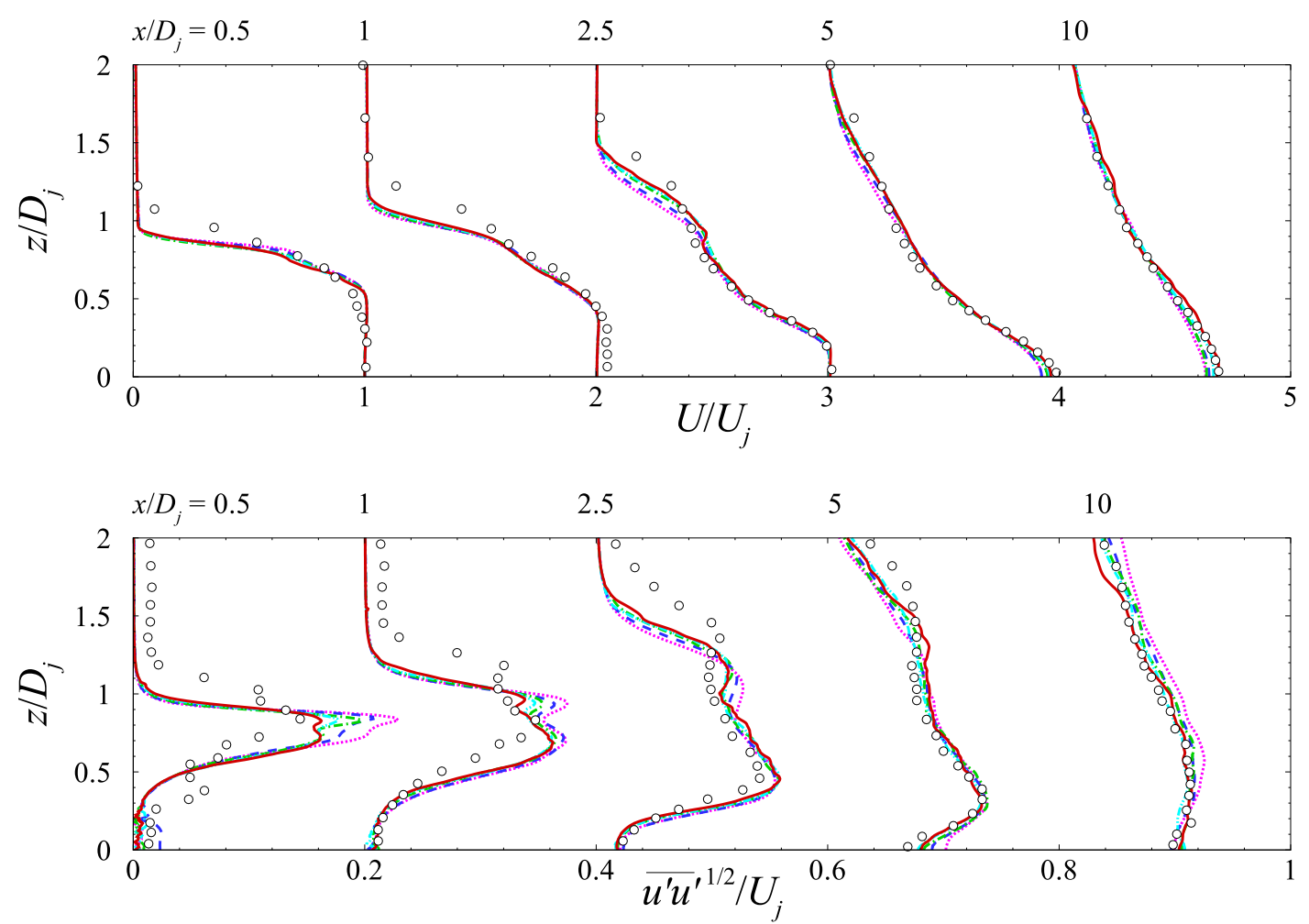

Figure 10: Chevron root cut plane. Top: velocity profiles. Bottom: normal stress profiles. Bridges and Wernet [67] $\bigcirc$, S6-05M (magenta dotted), S6-10M (blue dashed), S6-20M (green dash-dot), S6-40M (cyan dash-dot-dot), S6-80M (red solid).

outer region, possibly due to the absence of numerical inflow turbulence. The normal stress profiles are also shown, separated by an offset of 0.2 . As the flow through the chevron roots is strongly non-parallel, second order statistics are more challenging to predict. However, all grids are able to capture the two distinct peaks of the normal stress in the first streamwise locations. The grid refinement produces a reduction of the peak values, with the $80 \mathrm{M}$ grid yielding a remarkable agreement with the experiment even at $x / D_{j}=0.5$.

The different grid behaviour in the vicinity of the nozzle lip is captured in the energy spectra of axial velocity of Figure 11, where

$$
E_{11}(f)=\frac{1}{T} \int_{0}^{T} R_{11}(\tau) e^{-i 2 \pi f \tau} d \tau
$$

is the Fourier transform of the auto-correlation function

$$
R_{11}(\tau)=\frac{1}{T} \int_{0}^{T} u^{\prime}(t+\tau) u^{\prime}(t) d t
$$

With the reasonable assumption that the advection in a high speed jet is mostly due to the mean flow, 

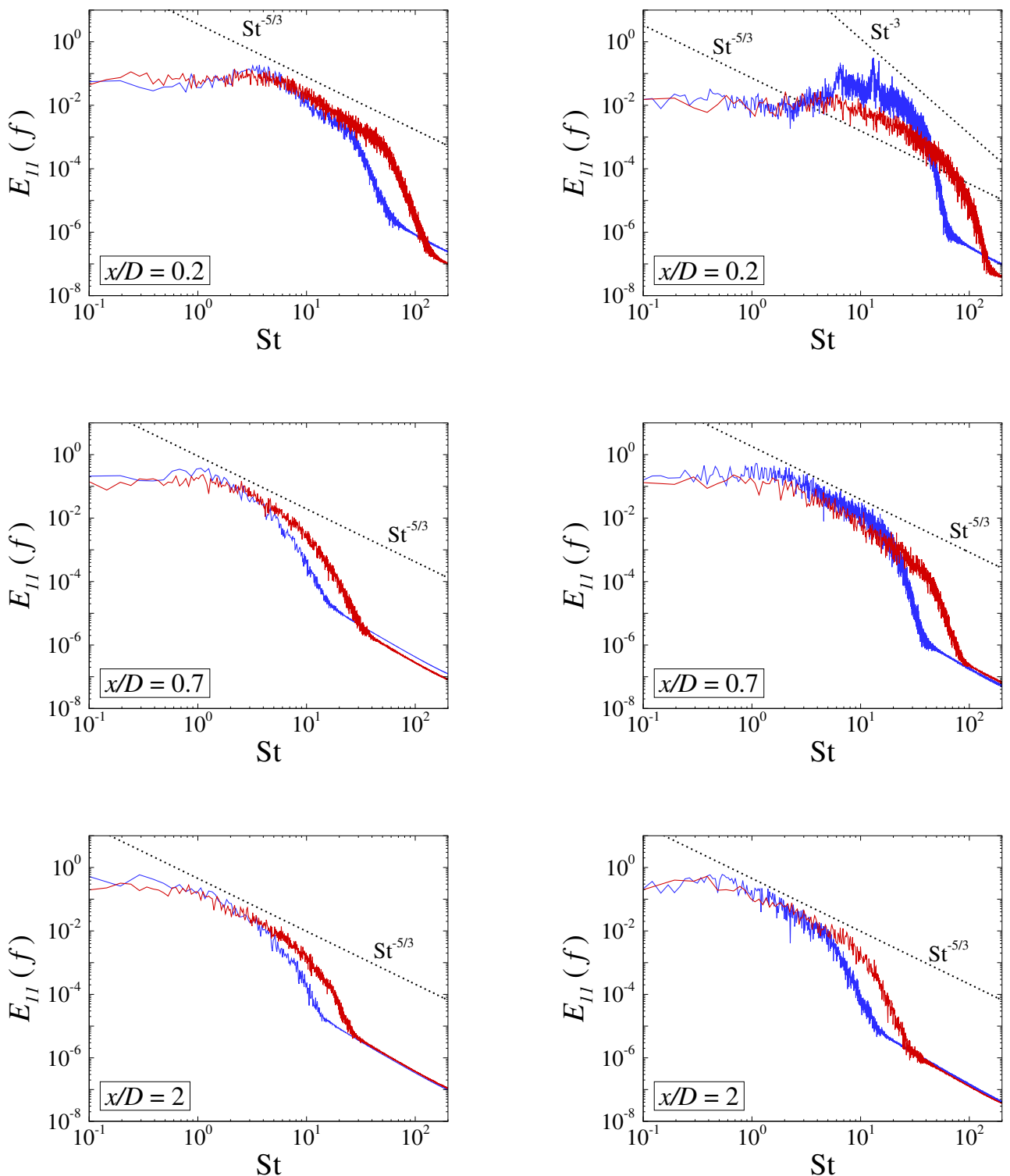

Figure 11: Energy spectra on the lipline of the round nozzle (left) and chevron nozzle tip (right); 10M grid (blue), 80M grid (red). 
Taylor's frozen turbulence hypothesis [68] can be used to compare time-resolved single-point measurements to well-known spatial behaviour in the wavenumber domain [69]. Figure 11 shows the spectra at three different locations along the lipline for cases R0-10M and R0-80M on the left; and the spectra along the lipline from a chevron tip for cases $\mathrm{S} 6-10 \mathrm{M}$ and $\mathrm{S} 6-80 \mathrm{M}$ on the right. At $x / D_{j}=0.2$ cases $\mathrm{R} 0-10 \mathrm{M}$ and $\mathrm{R} 0-80 \mathrm{M}$ present a spectrum in agreement with the typical $-5 / 3$ slope of $3 \mathrm{D}$ isotropic turbulence, evidence that the transition to three-dimensional turbulence has already occurred, even at this very early location. The coarser grid still presents minor discrepancies between the mid-range spectrum and the $-5 / 3$ slope, sign of a slightly delayed transition. Further downstream, at $x / D_{j}=0.7$ and $x / D_{j}=2$, the two spectra follow the $-5 / 3$ slope, with the only effect of grid refinement being the higher cut-off frequency in the finer case. The chevron cases present similar spectra. At $x / D_{j}=0.2$ the coarser grid shows a delayed transition, due to the relatively lower azimuthal resolution. The spectrum follows in part the $-5 / 3$ and in part the -3 slope, which means that the shear layer structures maintain some $2 \mathrm{D}$ coherence. Not far downstream, at $x / D_{j}=0.7$, the turbulence in the shear layer can already be considered three-dimensional for both grids, yielding a good agreement with the $-5 / 3$ slope. At $x / D_{j}=0.7$ and $x / D_{j}=2$ the grid refinement only increases the high cut-off frequency. Overall, grid refinement tends to break coherence and speed up the transition to more developed turbulence, but this effect is limited to locations immediately downstream from the nozzle lip. As will be shown in Section 4 , the grid refinement effect on the high cut-off frequency will produce a broadening of the spectra towards higher frequencies, with limited effect in the low and medium frequency range.

\section{Acoustic analysis}

\subsection{Near-field acoustics}

Jet noise studies usually focus on results in the far-field, where they can be directly compared to experimental data. However, far-field noise is the result of noise source generation and of sound propagation which are happening in the near-field. When using the FW-H approach, efforts need to be devoted to ensure that most sources are enclosed by the integration surface and that no excessive numerical dissipation occurs before the sound waves reach the surface.

Figure 12 shows pressure and vorticity contours for cases R0-10M and R0-80M. It is clear that a finer grid captures more detailed vortical structures and allows the propagation of higher-frequency (or shorter-wavelength) sound waves. The dominant radiation appears to be at low angles, around $30^{\circ}$ from the downstream direction, as expected for single-stream jets. 

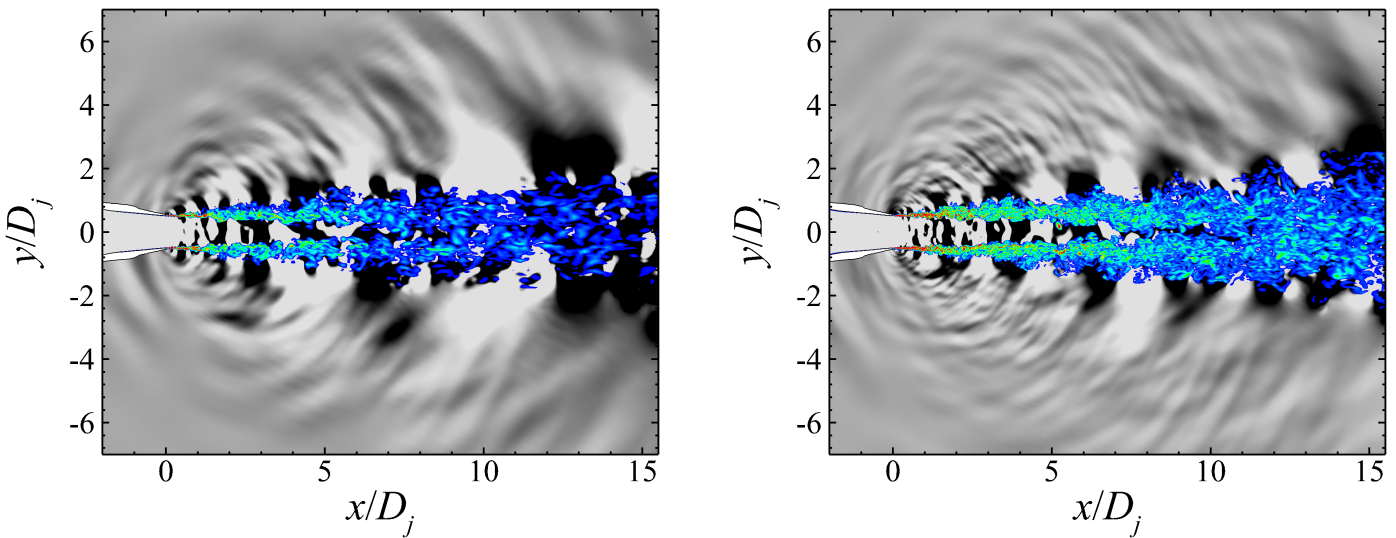

Figure 12: Near-field acoustic wave dilatation visualized by pressure contours (greyscale) and vorticity contours (rainbow). R0-10M on the left; R0-80M on the right. The color scales range up to the level of $16 U_{j} / D_{j}$ for vorticity, and from -100 to 100 $\mathrm{Pa}$ for pressure.

Since these waves present a broad range of frequencies, a Fourier analysis can help isolate the preferred directions [70] and assess the dissipative effect of grid coarsening. The temporal Fourier transform of the pressure fluctuation on the $x-y$ cut-plane, $\hat{p}(f)=\int_{-\infty}^{+\infty} p(t) e^{-2 \pi f t} d t$, is averaged over $1 / 12$ th octave bands at Strouhal numbers St $=0.3,0.6,1.0$ and 2.0, and the real part of its inverse transform is shown in Figure 13 for cases S6-10M and S6-80M. Lower-frequency waves $(\mathrm{St}=0.3)$ seem to originate around the end of the potential core $\left(x / D_{j} \approx 4\right)$, and radiate mainly at low polar angles. As frequency increases $(\mathrm{St}=0.6$, 1.0), the waves present additional acoustic beams at higher angles. At higher frequencies $(\mathrm{St}=2.0)$ sources spread along the whole mixing region, from the nozzle lip to beyond the end of the potential core, and sound radiates with no clear preferred direction.

The intensity of the waves, while they propagate, is clearly affected by their frequency. Since all the plots in Figure 13 share the same contour levels (same colour legend), it is easy to see qualitatively that different frequencies contain similar energy, at least up to a certain propagation distance. As will be shown in the following noise spectra, this is confirmed by experimental data for the chosen Strouhal numbers. The stronger dissipation at higher frequencies is due to the stretching of the grid, as can be clearly seen by comparing the two cases with 10 and $80 \mathrm{M}$ grid points. Figure 14 shows pressure fluctuations along the dash-dot lines depicted in Figure 13. The instantaneous nature of these plots does not allow for a rigorous analysis, nonetheless they are useful to underline a few aspects. For St $=1.0$ both grids present the expected $1 / r$ trend [66] up to a certain distance that seem to coincide with the location where the number of points per wavelength drops below $\approx 25$ (only one in every four data points is shown in Figure 14). Beyond that location $(r / D \approx 6$ for case S6-10M and $r / D \approx 8$ for case S6-80M, with $r / D=0$ on the jet centerline) the 

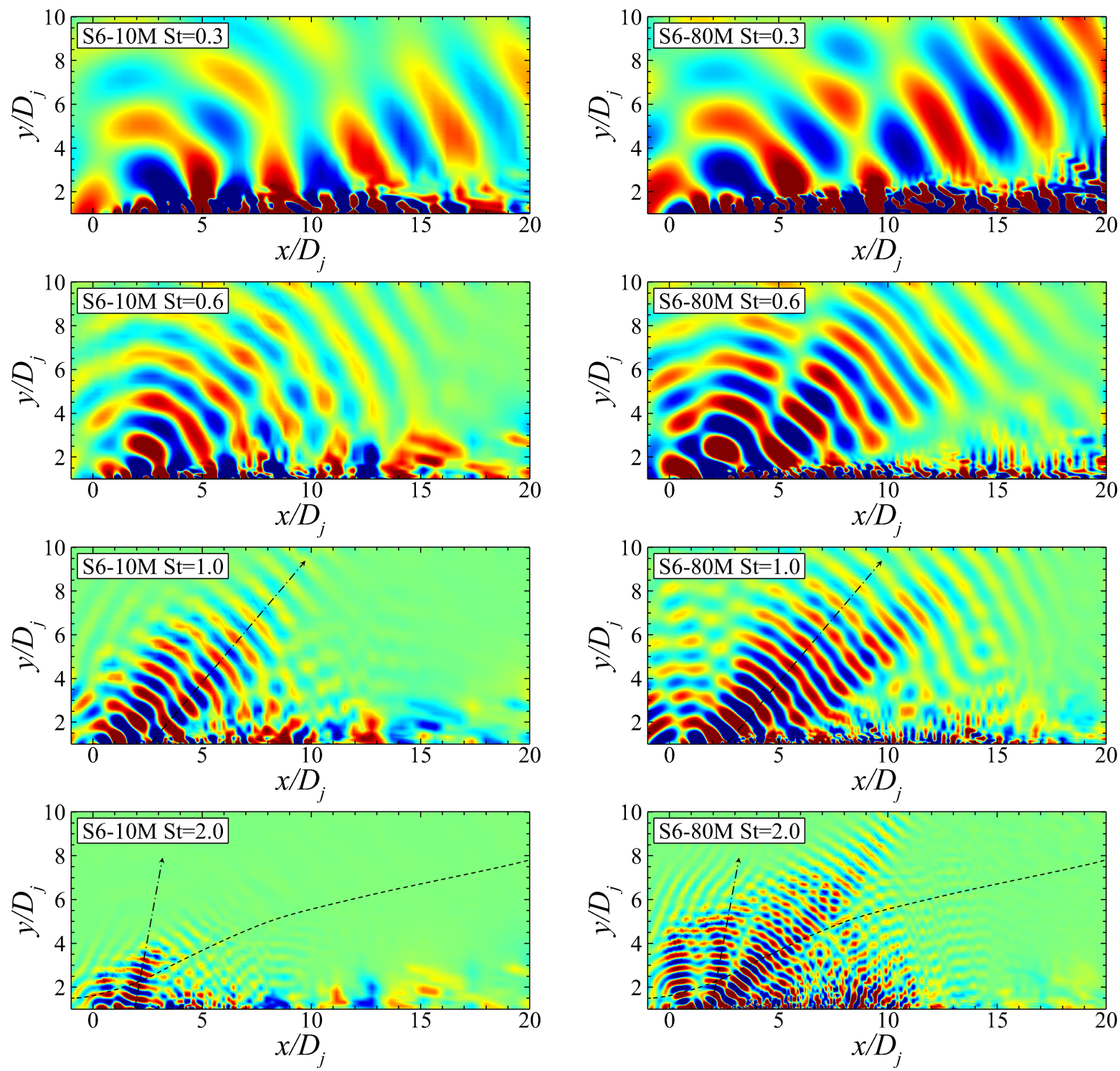

Figure 13: Real part of the filtered inverse Fourier transform of pressure fluctuations in the chevron tip plane for St $=0.3$, 0.6 , 1.0 and 2.0, for cases S6-10M and S6-80M. All color scales range from -5 to $5 \mathrm{~Pa}$. The dashed line is the FW-H surface, the dash-dot arrows are the directions used in Figure 14. 

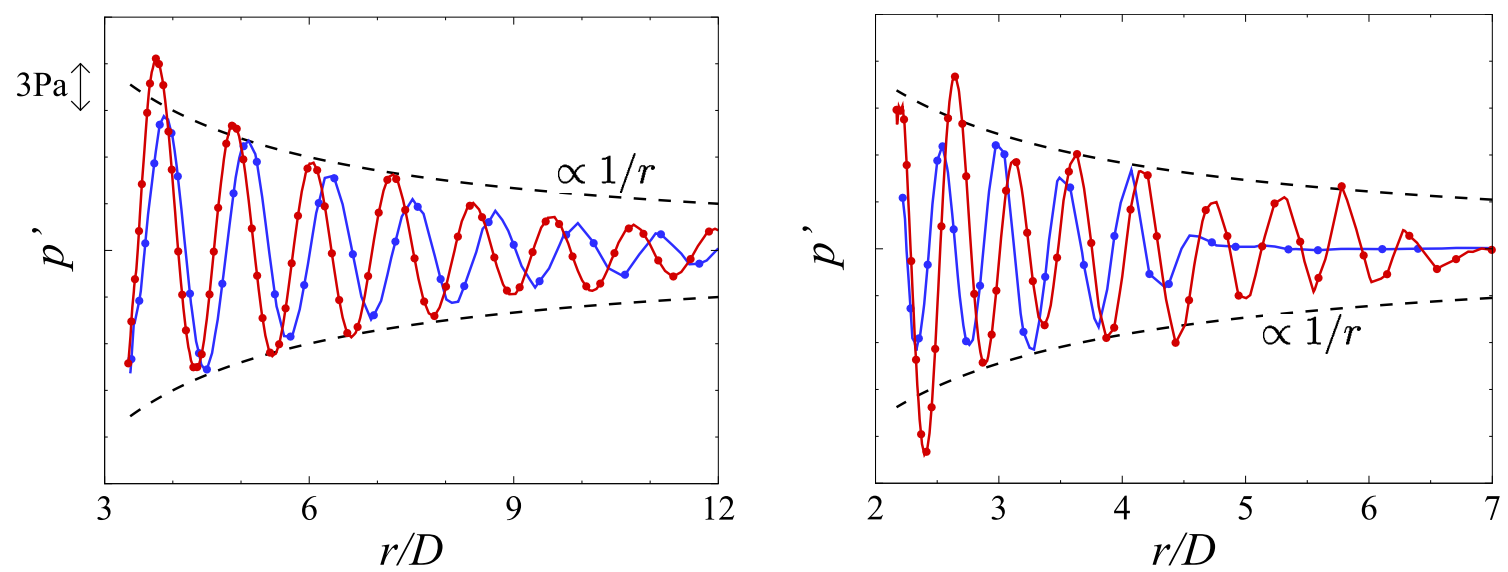

Figure 14: Pressure fluctuation along the dash-dot lines depicted in Figure 13 for St $=1.0$ (left) and St $=2.0$ (right). Only one every four data points is shown; S6-10M (blue), S6-80M (red).

energy contained in the wave gradually decreases due to numerical dissipation. For St $=2.0$ this effect is even more pronounced especially for the $10 \mathrm{M}$ grid, which is unable to sustain the correct amplitude. It is interesting to point out that if the excessive dissipation occurs before the waves reach the FW-H surface (dashed line in Figure 13), the far-field noise spectra will manifest an energy decrease. This means that the short distance propagation to the FW-H surface will produce a loss of high frequency information even if the corresponding noise sources are well resolved in the jet plume.

\subsection{Far-field acoustics}

Figure 15 shows a sketch of the FW-H surface with the sound observer positions. The surface is $25 D_{j}$ in axial length, and has diameters of around $3 D_{j}$ and $18 D_{j}$ at its ends. An surface-independence study is reported in Appendix B. The FW-H integrals are calculated for observers at $120 D_{j}$ from the nozzle exit centre at polar angles $\theta$, and averaged over equally spaced azimuthal positions.

The following FW-H sound spectra are compared to the experiments of Brown and Bridges [71]. The narrowband one-sided Power Spectral Density (PSD) is defined in its non-dimensional form as

$$
\mathrm{PSD}=10 \log _{10}\left(2 \frac{|\hat{p}|^{2}}{p_{0}^{2}} \frac{f_{0}}{\Delta f}\right)
$$

where $\hat{p}$ is the Fourier transform of the pressure fluctuation, $p_{0}$ is the reference pressure $2 \times 10^{-5} \mathrm{~Pa}$, and $\Delta f$ is the narrowband width, made non-dimensional by means of the reference frequency $f_{0}=1 \mathrm{~Hz}$.

Figures 16 and 17 show the PSD of the far-field sound at $\theta=120^{\circ}, 90^{\circ}, 60^{\circ}$ and $30^{\circ}$, for the round and serrated nozzle respectively. The spectra are filtered by averaging over one-third octave bands, in order to 


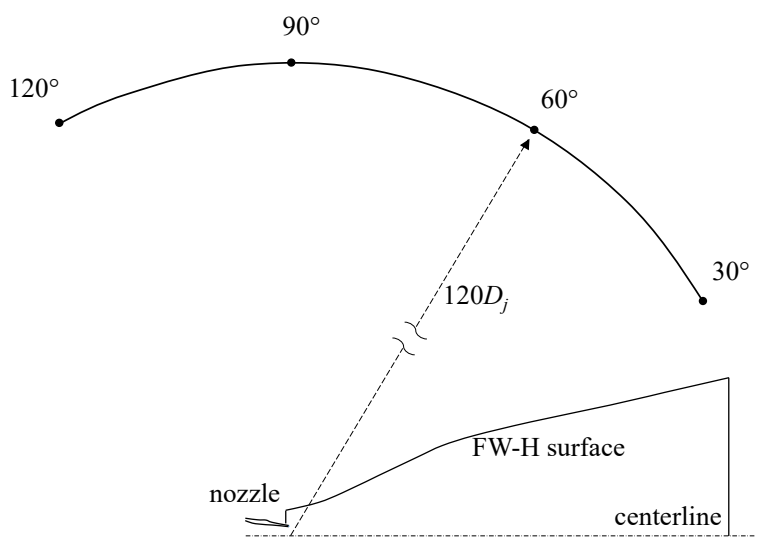

Figure 15: Representation of half of the FW-H surface profile and of the far-field sound observer position.

clarify the influence of the integration time and of the grid refinement. These spectra can be considered reliable above a certain frequency [20], which in this work was determined as $\mathrm{St}_{\min }=n D_{j} /\left(t_{\max } U_{j}\right)$, with $n=20$ to ensure sufficient convergence of the results. Hence all spectra obtained with an integration time of $300 t^{*}$ are displayed for $\mathrm{St}>\mathrm{St}_{\min } \approx 0.06$, while the $80 \mathrm{M}$ case $\left(50 t^{*}\right)$ is displayed for $\mathrm{St}>\mathrm{St}_{\min } \approx 0.4$.

For all observer angles the round jet presents a very good agreement with experimental results (Figure 16). The peak noise level is well captured by all grids, as is the decay rate before the high cut-off frequency $\mathrm{St}_{\max }$. For angles $\theta=60^{\circ}$ to $120^{\circ}$ the grid refinement produces a shift from $\mathrm{St}_{\max } \approx 1.5$ for case R0-05M to $\mathrm{St}_{\max } \approx 3.0$ for case R0-80M. For $\theta=30^{\circ}$ the effect is not as pronounced, with a shift from $\mathrm{St}_{\max }=0.7$ for case $\mathrm{R} 0-05 \mathrm{M}$ to $\mathrm{St}_{\max }>1.0$ for case $\mathrm{R} 0-80 \mathrm{M}$.

In the serrated case (Figure 17) the behaviour is similar to the round jet, with an even more noticeable shift in $\mathrm{St}_{\max }$ from $\approx 1.3$ for case $\mathrm{S} 6-05 \mathrm{M}$ to $\approx 3.0$ for case S6-80M for angles $\theta=60^{\circ}$ to $120^{\circ}$. The $\theta=30^{\circ}$ spectra do not show a noticeable dependence on grid refinement, and they all present a lower sound level at mid-to-high frequencies compared to the experiments, the cause of which has not been identified.

The collapse at low and mid frequencies of the presented spectra and the extension of the high frequency range due to grid refinement suggest that spectra from different grids could be combined to obtain a single broader spectrum, and this will be discussed in Section 4.3.

The spectra plotted in Figure 16 can be further analysed by means of azimuthal decomposition. The coefficients of a Fourier series in the azimuthal angle $\phi$ are

$$
C_{m}(t)=\frac{1}{2 \pi} \int_{-\pi}^{\pi} p(t, \phi) e^{i m \phi} d \phi
$$



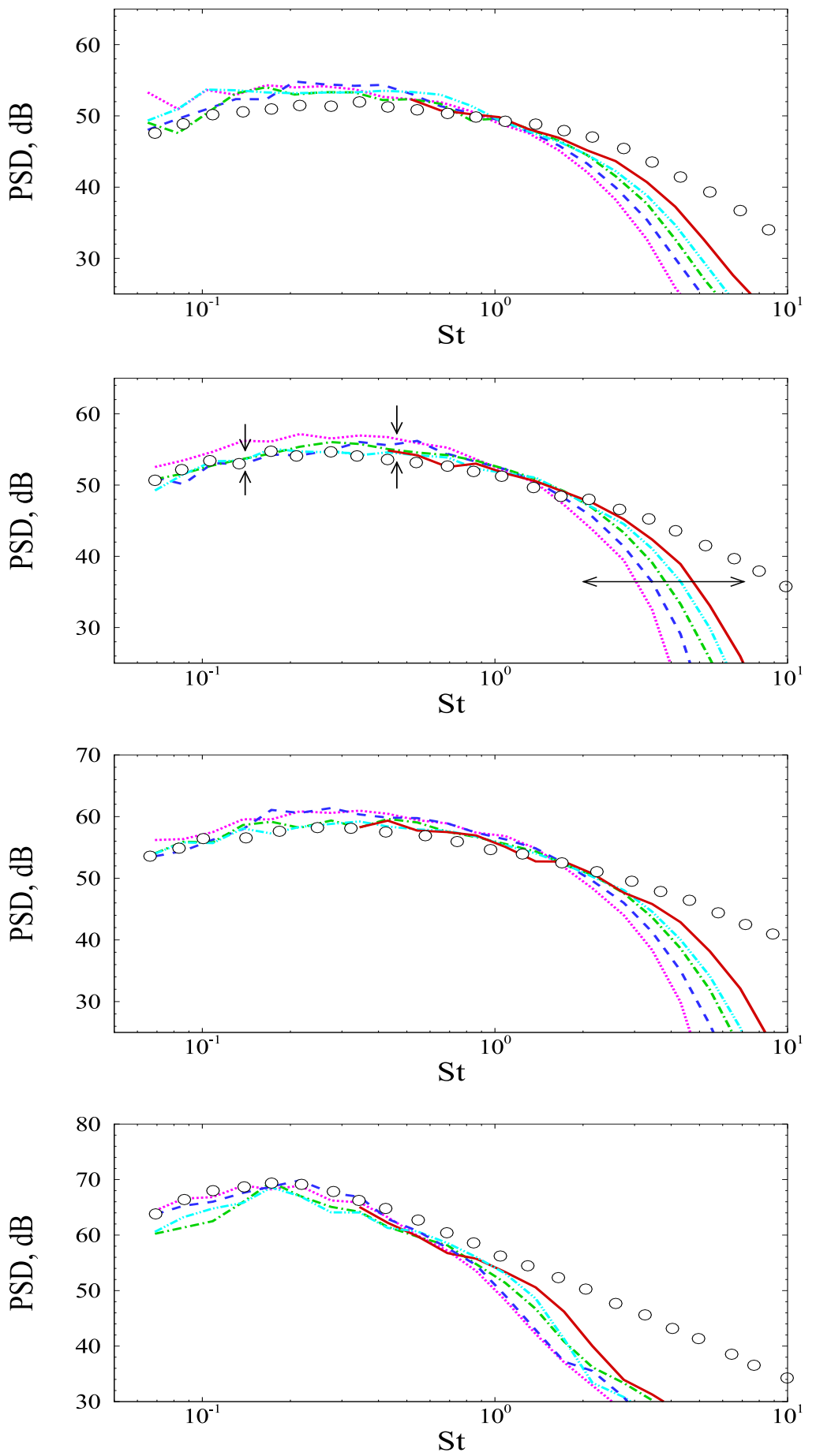

Figure 16: Far-field sound PSD at (from top to bottom) $\theta=120^{\circ}, 90^{\circ}, 60^{\circ}$ and $30^{\circ}$ for the round nozzle. Brown and Bridges [71] O, R0-05M (magenta dotted), R0-10M (blue dashed), R0-20M (green dash-dot), R0-40M (cyan dash-dot-dot), R0-80M (red solid). The arrows are an indication of the collapsing of the spectra at low and mid-frequencies and of their spreading towards higher frequencies due to grid refinement. 

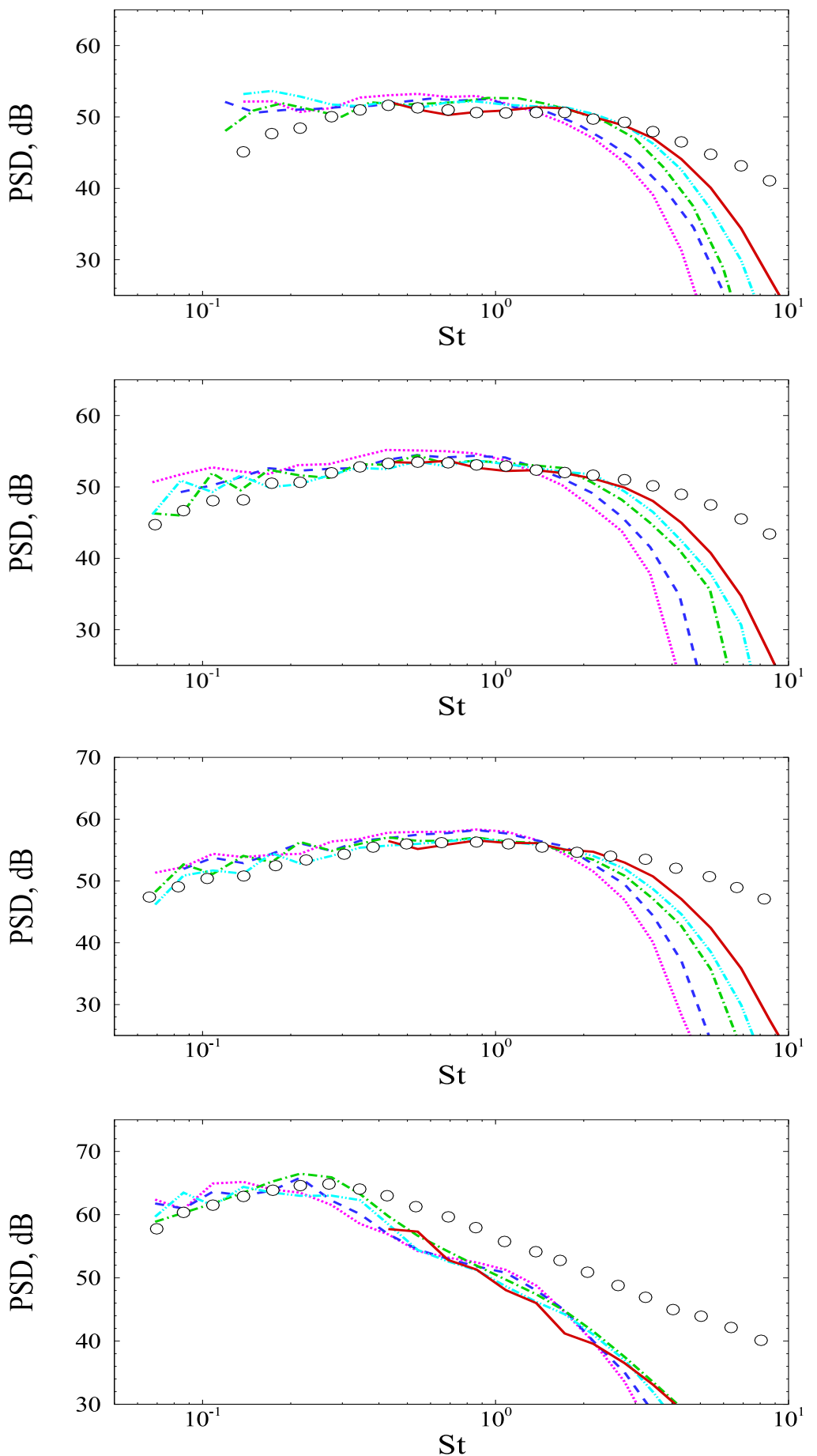

Figure 17: Far-field sound PSD at (from top to bottom) $\theta=120^{\circ}, 90^{\circ}, 60^{\circ}$ and $30^{\circ}$ for the chevron nozzle. Brown and Bridges [71] $\bigcirc$, S6-05M (magenta dotted), S6-10M (blue dashed), S6-20M (green dash-dot), S6-40M (cyan dash-dot-dot), S6-80M (red solid). 
Hence the azimuthal components of the pressure signal are $p_{0}(t)=C_{0}(t)$ and $p_{m}(t)=C_{-m}(t)+C_{m}(t)$ if $m \neq 0$. The importance of lower azimuthal mode events in noise generation was highlighted by several authors [72-74]. While it is expected that increasing the azimuthal resolution might result in higher modes being more energetic, it is important to verify that different azimuthal resolutions will equally capture the more dominant lower modes. For these calculations, 36 observer positions are used in the azimuthal direction. The modes analyzed below $(m=0,2,5,7)$ are chosen to highlight different trends.

Figure 18 compares the PSD at $\theta=30^{\circ}$ of four different azimuthal modes to the total signal from cases R0-10M and R0-80M. The axisymmetric mode $(m=0)$ is clearly predominant especially at the most energetic frequencies, evidence of a low azimuthal order organization at low polar angles. The influence of modes 1 (not shown) and 2 becomes noticeable only beyond $\mathrm{St}=1$. Modes 5 and 7 show an even lower overall energy. Interestingly, here the peak levels seem to vary significantly with the mesh refinement, as the higher resolution allows for more energy to be organized at these higher azimuthal modes. All modes present a shift in cut-off frequency due to mesh refinement, as already discussed for the total signal.

Figure 19 shows the PSD of the same modes at $\theta=90^{\circ}$. Modes 0 to 2 are the strongest ones, with similar energy levels, showing the same behaviour of the total signal with a higher cut-off frequency in the finer-grid case. From mode 3 (not shown) to 5 the overall energy decreases, especially at lower frequencies. This confirms the link between lower azimuthal mode events and far-field noise at high polar angles. The two grids are in good agreement until mode 7 , where the finer grid clearly captures a higher energy content than the coarser one at all frequencies. However, the energy content is now negligible compared to lower modes along most of the spectrum. This means that higher resolution will be able to better capture the energy content of high frequencies and high azimuthal modes, but with a small effect on the integrated overall sound pressure level, as defined by

$$
\text { OASPL }=10 \log _{10} \int_{0}^{\infty}\left(2 \frac{|\hat{p}|^{2}}{p_{0}^{2}} \frac{1}{\Delta f}\right) d f
$$

Figures 20 and 21 show the OASPL at different polar angles for the total signal and for single azimuthal modes, with the round and chevron nozzle respectively. The total signal agrees well with the measurements of Tanna [41] and of Brown and Bridges [71], with no noticeable dependence on the grid size. In the sideline direction (around $90^{\circ}$ ) modes 1 and 2 have similar energetic levels as the axisymmetric mode $(m=0)$, as already shown in Figure 19. In fact, modes 1 and 2 are the most energetic for $50^{\circ}<\theta<100^{\circ}$. For lower angles (around $30^{\circ}$ ) the pressure field is dominated by the axisymmetric mode, and modes higher than 2 become negligible, as anticipated in Figure 18. These results are in agreement with experimental [75] and 

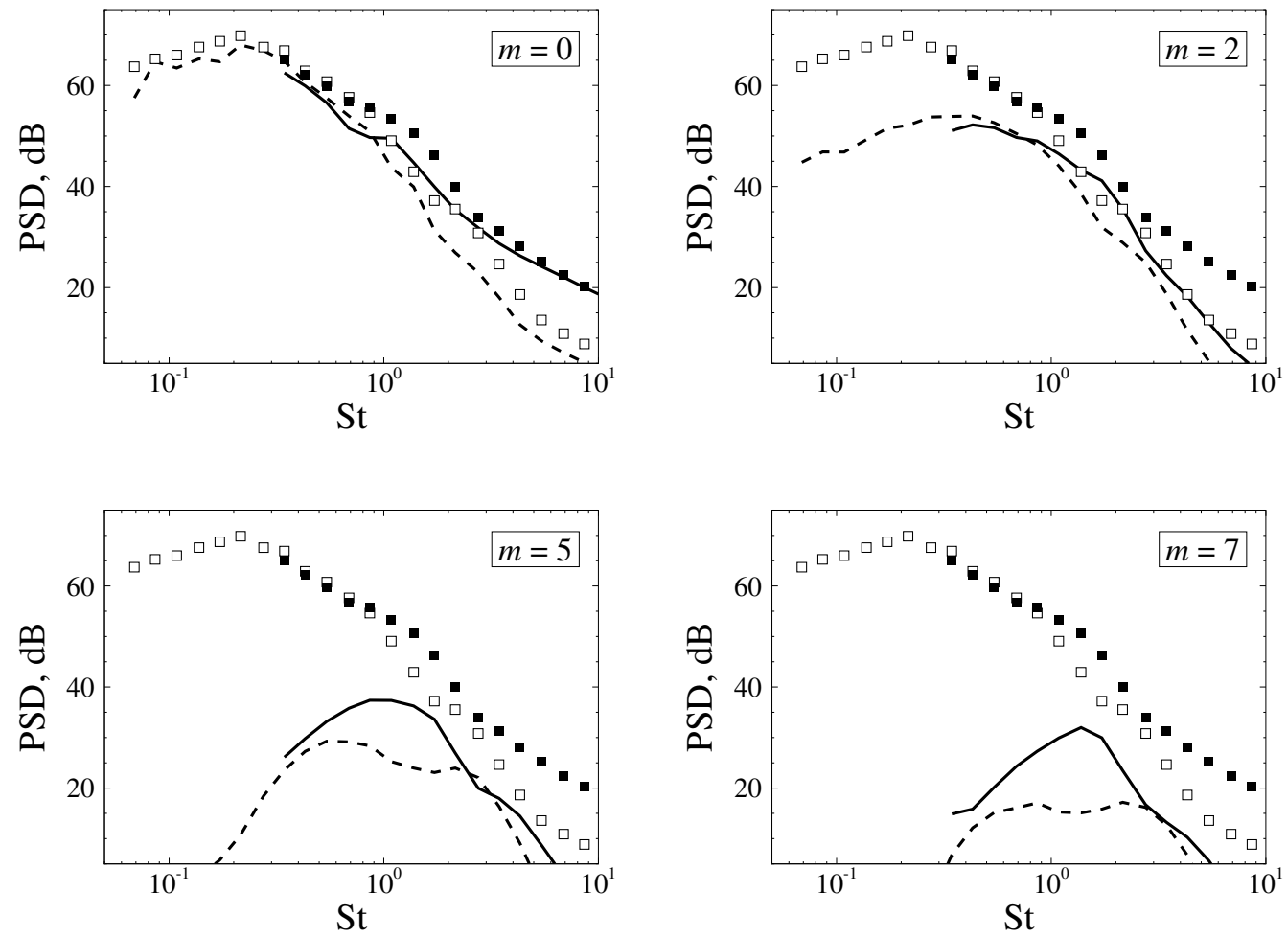

Figure 18: PSD of far-field azimuthal modes at $\theta=30^{\circ}$ for the round nozzle. Total signal, R0-10M $\square$; total signal, R0-80M single mode $m$, R0-10M (- - ); single mode $m$, R0-80M (-).

numerical $[72,73]$ studies. The effect of the grid refinement becomes noticeable for higher azimuthal modes: mode 5 in Figure 20 shows the same peak OASPL for the two grids, but its PSD level drops below 70dB for $\theta<40^{\circ}$ with the $10 \mathrm{M}$ grid, and for $\theta<20^{\circ}$ with the $80 \mathrm{M}$ grid; mode 7 follows a similar trend, but with a lower pressure level at all angles, as already anticipated in Figures 18 and 19. Similarly, in Figure 21 modes 5 shows a slight dependency from the grid refinement at all angles, which becomes more evident in mode 7 . Azimuthal decomposition thus confirms that lower modes can be accurately captured with coarser grids, while finer grids can be used to add high-mode information.

\subsection{Further discussion on a potential spectrum-broadening approach}

The results presented in the previous sections show that spectra from different grids collapse in the low and medium frequency range, as even relatively coarse grids can yield accurate results, while finer simulations widen the resolved spectral range towards high frequencies and high modes. This conclusion can form the basis for an efficient approach to obtain a more complete and broader spectrum, covering both the high- and 

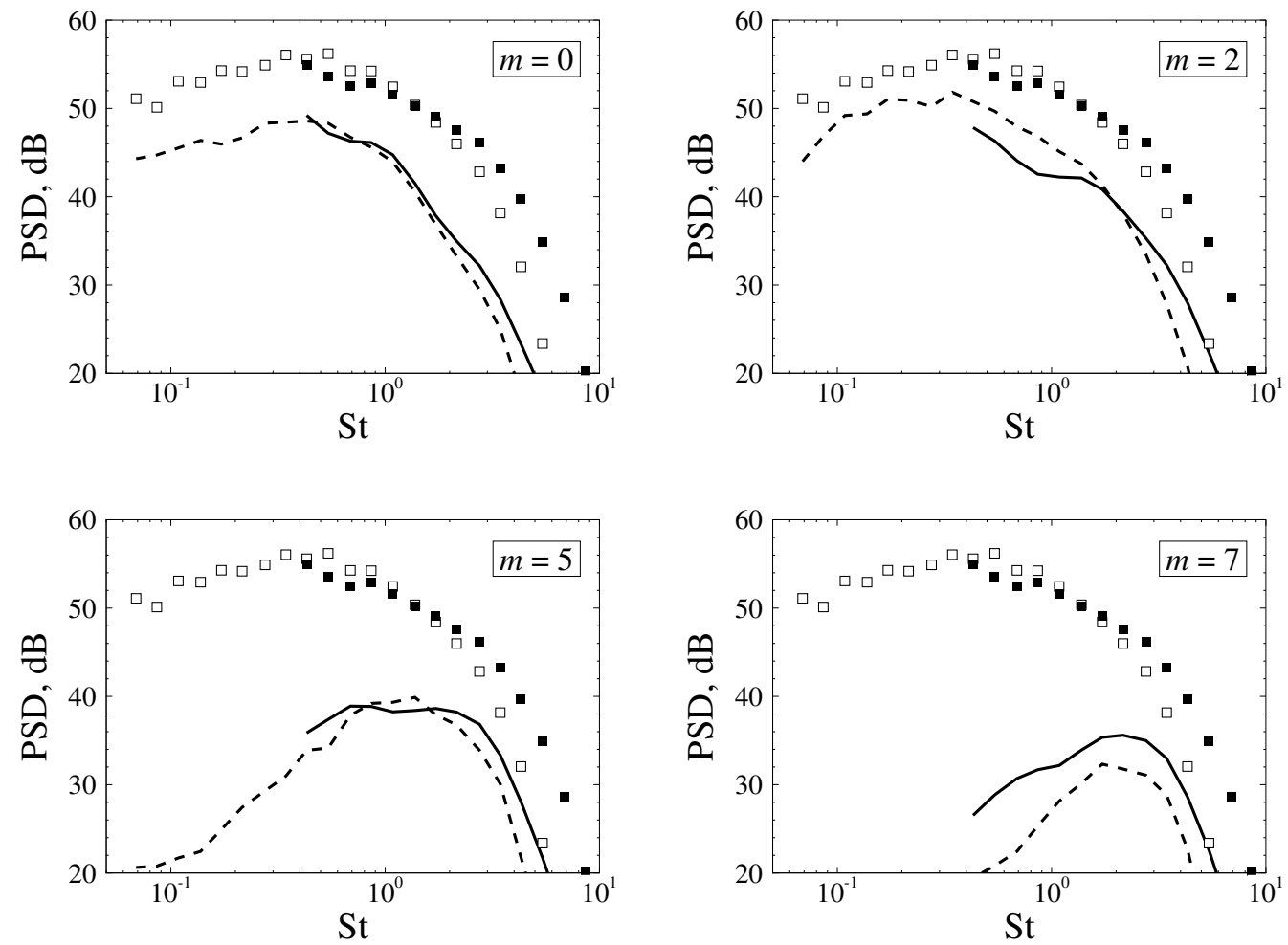

Figure 19: PSD of far-field azimuthal modes at $\theta=90^{\circ}$ for the round nozzle. Total signal, R0-10M $\square$; total signal, R0-80M single mode $m$, R0-10M (- ); single mode $m$, R0-80M ( - ).
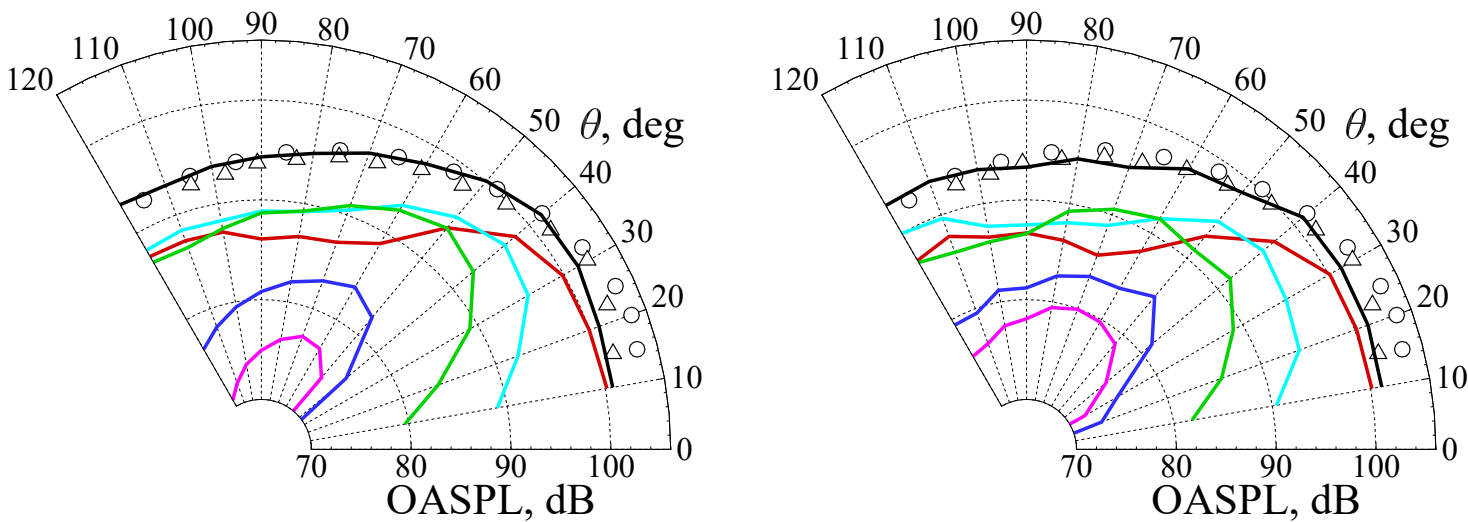

Figure 20: OASPL of far-field azimuthal modes for the round nozzle; R0-10M on the left, R0-80M on the right. Tanna [41] $\triangle$, Brown and Bridges [71] $\bigcirc$, total signal (black line), mode 0 (red), mode 1 (cyan), mode 2 (green), mode 5 (blue), mode 7 (magenta). 

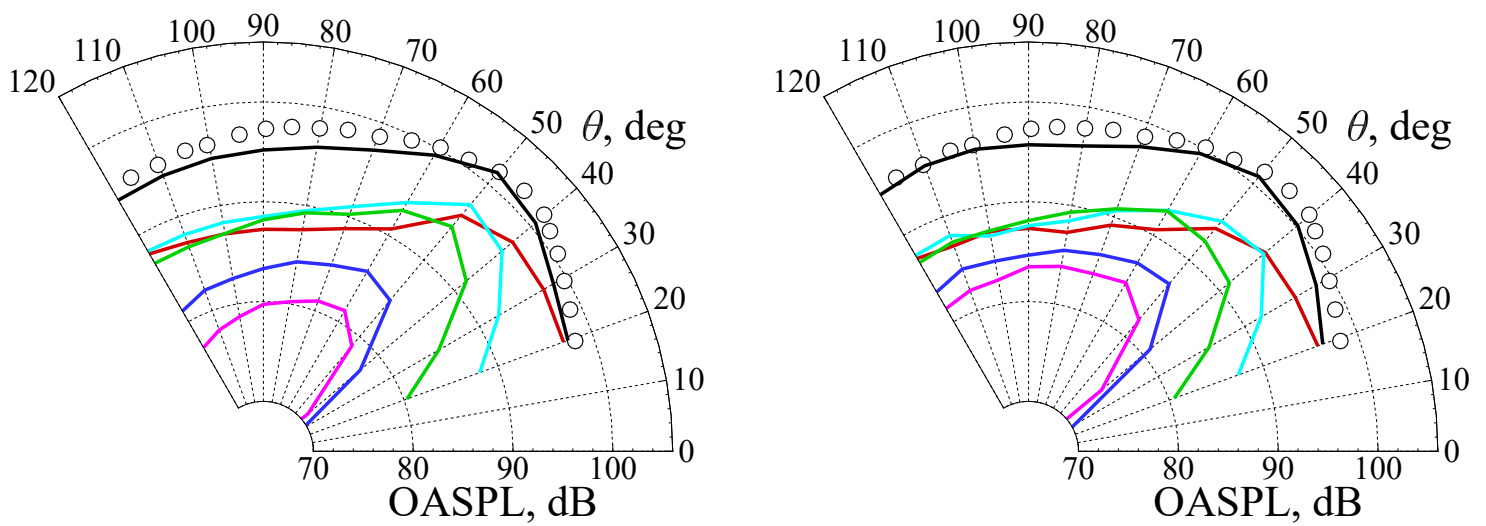

Figure 21: OASPL of far-field azimuthal modes for the chevron nozzle; S6-10M on the left, S6-80M on the right. Brown and Bridges [71] $\bigcirc$, total signal (black line), mode 0 (red), mode 1 (cyan), mode 2 (green), mode 5 (blue), mode 7 (magenta).

low-end of the Strouhal number range, by combining noise spectra from simulations with different spatial and temporal resolution.

A similar concept was previously studied by Bodard et al. [76], but with different methodologies for two parts of the spectrum, namely an LES approach for the low- and mid-range frequency, in conjunction with high frequency fine-scale mixing noise obtained from Tam and Auriault's model [77]. In our argument here the choice of using the same LES/FW-H approach but with different grid resolutions can ensure consistency in the overlapping region. The added computational cost of the fine-grid simulation is only required to resolve the high frequency components and therefore can be minimized by optimal choices of grid sizes, sampling time, and overlap/cut-off frequencies.

From an a-priori stand point, especially when there is a lack of experimental data, a good understanding of the relation between grid spacing and cut-off frequency $\mathrm{St}_{\max }$ needs to be first established. A first estimate for $\mathrm{St}_{\max }$ can be derived from the grid cell sizes in proximity of the $\mathrm{FW}-\mathrm{H}$ surface, with the requirement of 25 points per wavelength, as discussed in Section 4.1. The analysis of the Fourier-decomposed pressure waves, e.g. in Figure 13, can yield a more accurate estimate of $\mathrm{St}_{\max }$, without the need for comparison with experiments. Depending on the position of the FW-H surface, it is possible to identify the frequencies that will reach the surface and those that will not. As shown in Figure 13, the 10M grid seems to dampen most acoustic waves for $\mathrm{St} \geqslant 2$ before they reach the $\mathrm{FW}-\mathrm{H}$ surface, especially the ones generated near the end of the potential core, where the grid spacing is less favourable. For a given cut-off frequency $\mathrm{St}_{\max } \sim 3$, an $80 \mathrm{M}$ grid is necessary with the present grid strategy, with halved time step $d t$ compared to the $10 \mathrm{M}$ case to maintain numerical stability and accuracy. This finer case will therefore cost roughly 16 times more than the coarser case per physical time unit $t^{*}$. With a typical integral time for $\mathrm{FW}-\mathrm{H}$ extrapolation of at least $50 t^{*}$, 
the lowest achievable Strouhal number $\mathrm{St}_{\min }$ will be around 0.4, as discussed in Section 4.2. Hence, a 10M grid, with a cut-off frequency St $\sim 2$, allows for a spectrum overlap with the $80 \mathrm{M}$ case. The computation time of the $10 \mathrm{M}$ case running for $300 t^{*}, T_{10 M, 300}$, is almost three times lower than that of the $80 \mathrm{M}$ case

running for $50 t^{*}, T_{80 M, 50}$ (see Table 2 ). This results in the overall running cost $T_{t o t}=T_{80 M, 50}+T_{10 M, 300}$ being less than one quarter of the time $T_{80 M, 300}$ that would be required to cover the same spectrum with one fine grid solution.

The grid strategy applied in this paper was chosen to ensure consistency in the successive refinement. For the discussed approach to be viable for even higher cut-off frequencies, as is typically required in an industrial context, a more carefully designed zonal grid refinement is necessary, to yield broader noise spectra for the same global grid size.

\section{Conclusions}

Eddy-resolving simulations have been carried out for subsonic turbulent jets from an axisymmetric nozzle and a serrated nozzle, with successively refined grids. The test cases are specifically chosen so that comparisons with experimental data can be made. Favourable agreement has been achieved in the near-field jet turbulence statistics including the mean and fluctuating quantities. In particular, the predictions obtained for the round nozzle with the finer grids show excellent agreement with the experiments on potential core length, despite this often being challenging as underprediction is typically observed in the literature.

A Fourier analysis is applied to the near-field noise propagation in respect to grid resolution. This allows to identify the directional behaviour of the sound waves and is particularly useful when assessing dissipation effects of grid coarsening. The analysis of the dampened high-frequency components over the relatively short-distance to the FW-H surface has shown to be a useful indicator to gain a priori knowledge of the far-field cut-off frequency.

The analysis of far-field sound pressure levels and azimuthal modes is further performed and several observations can be made: a) different noise frequency ranges and modes are affected in a different way by the grid resolution; b) sound spectra tend to collapse in the low and medium frequency range; c) a spreading of the spectra towards higher frequencies with the increasing resolution is clearly identifiable.

A preliminary discussion and running cost budget are presented for a potential spectrum-broadening approach, based on the combination of multiple spectra from successively refined grids. 


\section{Acknowledgements}

The authors acknowledge the support from UK's Engineering and Physical Sciences Research Council (EPSRC), including the computer time allocation units on the Tier-1 machine ARCHER in conjunction with the additional units provided via the UK Turbulence Consortium (under grant number EP/L000261). We also thank HPC Midlands and Loughborough University for CPU time and IT support.

\section{Appendix A. Grid points distribution}

The point distribution along the curvilinear coordinate $x$ follows:

$$
x(\xi)=s(\xi)\left(x_{1}-x_{0}\right)+x_{0} \quad \xi \in[0, N]
$$

where $x_{0}$ and $x_{1}$ are the edge ends, $\xi$ is the grid point index, $N$ is the number of grid points, and $s(\xi)$ is a tanh-based stretching function defined as follows [78]:

$$
s(\xi)=\frac{u(\xi)}{A+(1-A) u(\xi)}
$$

where

$$
\begin{array}{ll}
u(\xi)=\frac{1}{2}+\frac{\tanh [\Delta y(\xi / N-1 / 2)]}{2 \tanh [\Delta y / 2]} & \text { if } B>1 \\
u(\xi)=\frac{1}{2}+\frac{\tan [\Delta z(\xi / N-1 / 2)]}{2 \tan [\Delta z / 2]} & \text { if } B<1 \\
u(\xi)=\xi / N[1+2(B-1)(\xi / N-1 / 2)(1-\xi / N)] & \text { if } B \approx 1
\end{array}
$$

Here $A=\sqrt{s_{0} / s_{1}}, B=\sqrt{s_{0} s_{1}}, s_{0}=\left(x_{1}-x_{0}\right) /\left(\Delta x_{0} N\right)$ and $s_{1}=\left(x_{1}-x_{0}\right) /\left(\Delta x_{1} N\right) . \Delta x_{0}$ and $\Delta x_{1}$ are fixed spacings at each end of the line, while $\Delta y$ and $\Delta z$ are defined implicitly by $B=\sinh \Delta y / \Delta y$ and $B=\sin \Delta z / \Delta z$. Figure A1 gives a demonstration of spacing and growth rate when doubling the number of cells along an edge with the current strategy (squares), compared to a mid-cell "naive" splitting of the initial cells (crosses). The rigorousness of our method ensures smooth evolution of spacing and growth rate and therefore a fair comparison between grids, with a consistent and well-defined refinement at every location.

\section{Appendix B. FW-H surface-independence study}

The FW-H surface is based on the 'S3' surface of Shur et al. [64]. A validation of the FW-H procedure was carried out by comparing the signal propagated to a near-field observer located at radial distance of $2 D$, 

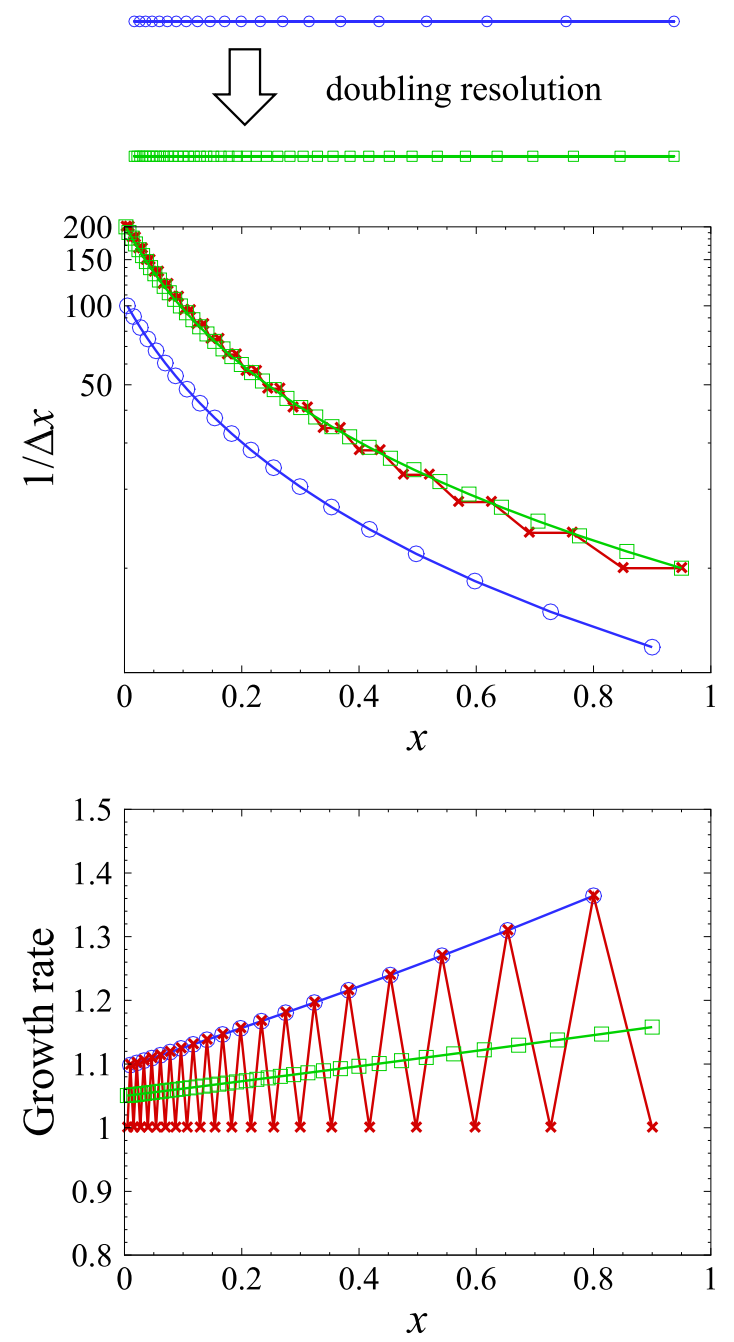

Figure A1: Sketch of resolution doubling of a grid edge (top), and the resulting spacing (middle) and growth rate (bottom). Original (blue circles), mid-cell sub-splitting (red crosses), and current refinement strategy with tanh clustering (green squares).

with the signal detected by a probe directly from the LES simulation. Figure B1 shows the agreement for a $90^{\circ}$ near-field PSD from case S6-10M.

In order to test the sensitivity of the far-field noise to the surface design, a series of simulations were performed for case R0-10M with a set of different surfaces, shown in Figure B2, with slightly different radial (from R1 to R4) and axial extent (from L1 to L3). Following Spalart and Shur [19], the surfaces are closed at the outflow disk. The mesh was designed to be as uniform as possible along the different surfaces, for the results not to be affected by the grid stretching.

Figure B3 compares the far-field raw sound PSD at $\theta=30^{\circ}$ and $90^{\circ}$, for all $\mathrm{FW}-\mathrm{H}$ surfaces. All spectra 


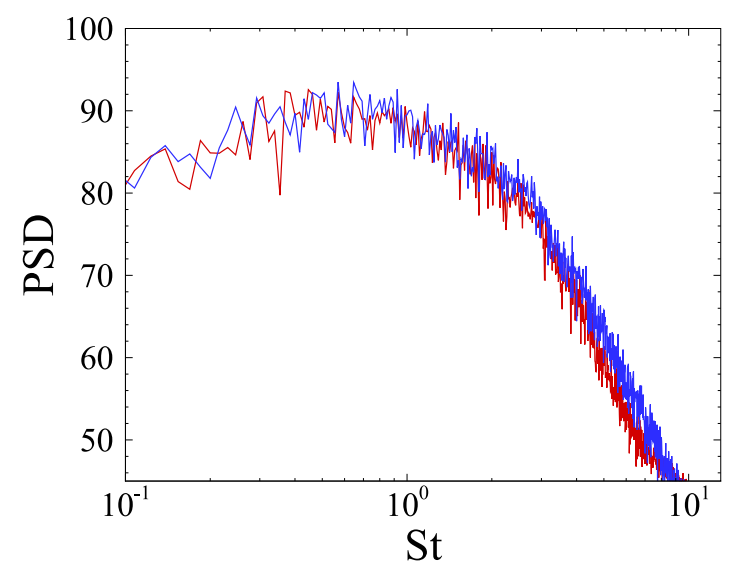

Figure B1: Comparison of the near-field $90^{\circ}$ power spectral density for case S6-10M, from an LES probe (red) and from FW-H (blue).

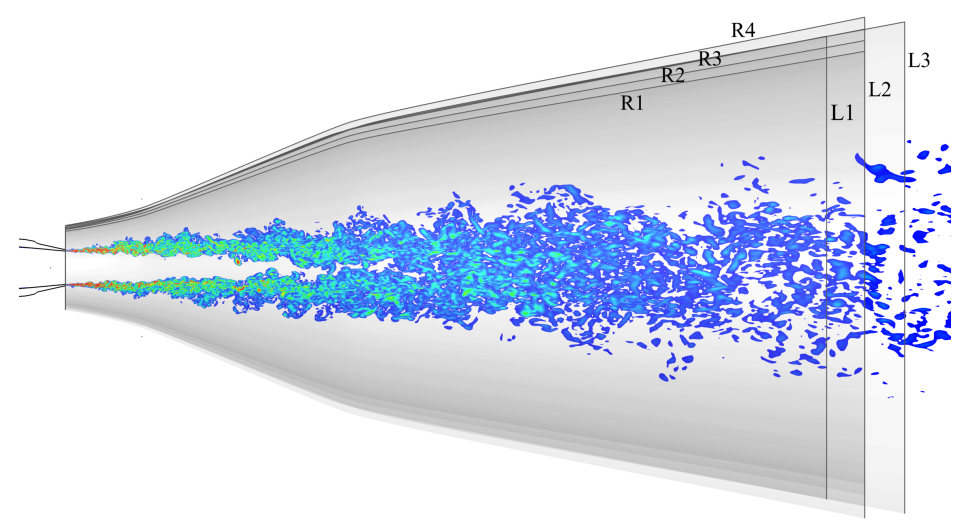

Figure B2: FW-H surfaces with different radial (from R1 to R4) and axial extent (from L1 to L3). The jet extent is illustrated by vorticity contours.

show very good agreement, with only a slight discrepancy at $\theta=30^{\circ}$ for different radial extents, but only for frequencies beyond the high cut-off frequency. This independence from the surface extent, and in particular from the location of the closing disk, is an important confirmation of the consistency of the chosen method. For all the other simulations in the present work, surface R3-L2 is used.

\section{References}

\section{References}

[1] High Level Group on Aviation Research, Flightpath 2050 Europe's Vision for Aviation, Technical Report, European Commission, 2011. 

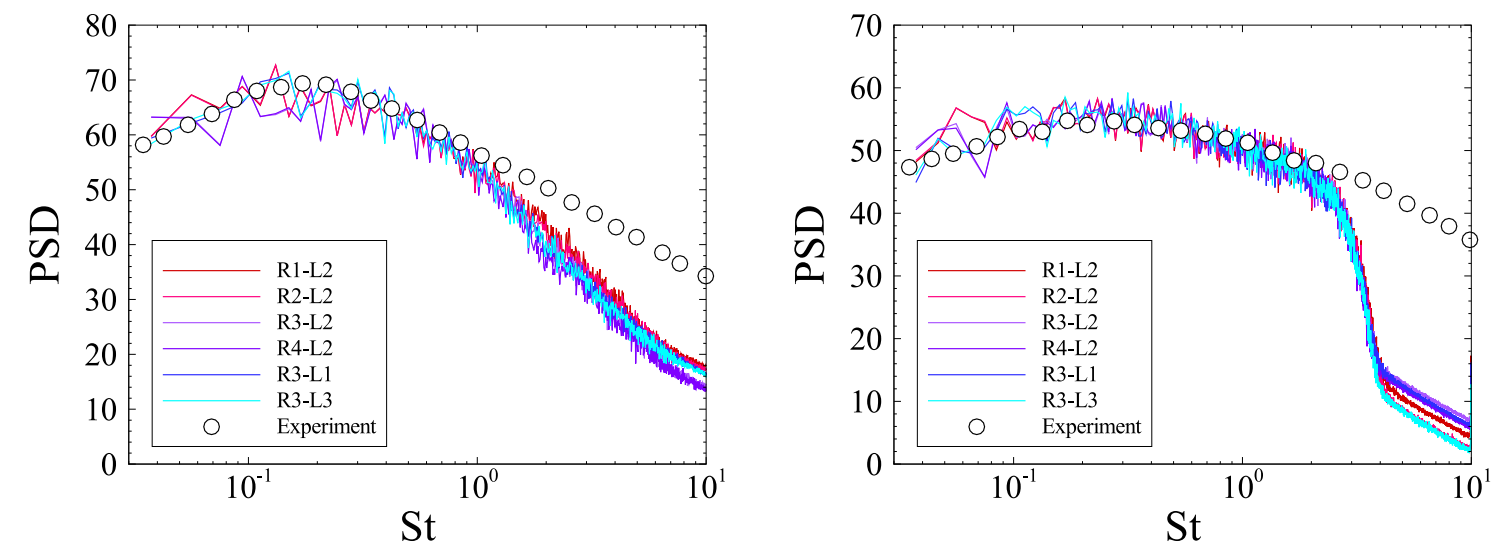

Figure B3: Far-field raw sound PSD at $\theta=30^{\circ}$ (left) and $90^{\circ}$ (right) for all FW-H surfaces, compared to the experimental data of Brown and Bridges [71].

[2] M. J. Lighthill, On Sound Generated Aerodynamically. I. General Theory, Proceedings of the Royal Society A: Mathematical, Physical and Engineering Sciences 211 (1952) 564-587.

[3] M. J. Lighthill, On Sound Generated Aerodynamically. II. Turbulence as a Source of Sound, Proceedings of the Royal Society A: Mathematical, Physical and Engineering Sciences 222 (1954) 1-32.

[4] C. K. Tam, Jet Noise: Since 1952, Theoretical and Computational Fluid Dynamics 10 (1998) 393-405.

[5] D. J. Bodony, S. K. Lele, Current Status of Jet Noise Predictions Using Large-Eddy Simulation, AIAA Journal 46 (2008) $364-380$.

[6] C. Bogey, C. Bailly, D. Juvé, Noise Investigation of a High Subsonic, Moderate Reynolds Number Jet Using a Compressible Large Eddy Simulation, Theoretical and Computational Fluid Dynamics 16 (2003) 273-297.

[7] D. J. Bodony, S. K. Lele, On using large-eddy simulation for the prediction of noise from cold and heated turbulent jets, Physics of Fluids 17 (2005) 085103.

[8] C. Bogey, C. Bailly, Computation of a high Reynolds number jet and its radiated noise using large eddy simulation based on explicit filtering, Computers \& Fluids 35 (2006) 1344-1358.

[9] C. Bogey, S. Barré, D. Juvé, C. Bailly, Simulation of a hot coaxial jet: Direct noise prediction and flow-acoustics correlations, Physics of Fluids 21 (2009) 035105.

[10] M. Zhu, C. Pérez Arroyo, A. Fosso Pouangué, M. Sanjosé, S. Moreau, Isothermal and heated subsonic jet noise using large eddy simulations on unstructured grids, Computers \& Fluids 171 (2018) 166-192.

[11] G. A. Brès, P. Jordan, V. Jaunet, M. Le Rallic, A. V. G. Cavalieri, A. Towne, S. K. Lele, T. Colonius, O. T. Schmidt, Importance of the nozzle-exit boundary-layer state in subsonic turbulent jets, Journal of Fluid Mechanics 851 (2018) 83-124.

[12] G. Kirchhoff, Zur Theorie der Lichtstrahlen, Annalen der Physik 254 (1883) 663-695.

[13] J. E. FFowcs Williams, D. L. Hawkings, Sound Generation by Turbulence and Surfaces in Arbitrary Motion, Philosophical Transactions of the Royal Society A: Mathematical, Physical and Engineering Sciences 264 (1969) 321-342.

[14] A. Biancherin, N. Lupoglazoff, G. Rahier, F. Vuillot, Comprehensive 3d Unsteady Simulations of Subsonic and Supersonic Hot Jet Flow-Fields: Part 2: Acoustic Analysis, AIAA 2002-2600.

[15] P. J. Morris, L. N. Long, T. E. Scheidegger, S. Boluriaan, Simulations of Supersonic Jet Noise, International Journal of 
Aeroacoustics 1 (2002) 17-41.

[16] A. Uzun, A. Lyrintzis, G. Blaisdell, Coupling of Integral Acoustics Methods with LES for Jet Noise Prediction, AIAA 2004-0517.

[17] M. Shur, P. Spalart, M. Strelets, Noise prediction for increasingly complex jets. Part I: Methods and tests, International Journal of Aeroacoustics 4 (2005) 213-246.

[18] M. Shur, P. Spalart, M. Strelets, Noise prediction for increasingly complex jets. Part II: Applications, International Journal of Aeroacoustics 4 (2005) 247-266.

[19] P. R. Spalart, M. L. Shur, Variants of the Ffowcs Williams - Hawkings Equation and Their Coupling with Simulations of Hot Jets, International Journal of Aeroacoustics 8 (2009) 477-491.

[20] S. Mendez, M. Shoeybi, S. Lele, P. Moin, On the use of the Ffowcs Williams-Hawkings equation to predict far-field jet noise from large-eddy simulations, International Journal of Aeroacoustics 12 (2013) 1-20.

[21] H. Xia, P. G. Tucker, S. Eastwood, Large-eddy simulations of chevron jet flows with noise predictions, International Journal of Heat and Fluid Flow 30 (2009) 1067-1079.

[22] M. Angelino, H. Xia, M. A. Moratilla-Vega, G. J. Page, Far-field Noise Prediction of Round and Serrated Jets with Increasingly Refined Grids, AIAA 2016-3047, Lyon, France.

[23] N. Saiyed, K. Mikkelsen, J. Bridges, Acoustics and thrust of separate-flow exhaust nozzles with mixing devices for high-bypass-ratio engines, AIAA 2000-1961.

[24] J. Bridges, C. Brown, Parametric Testing of Chevrons on Single Flow Hot Jets, AIAA 2004-2824.

[25] B. Callender, E. J. Gutmark, S. Martens, Far-Field Acoustic Investigation into Chevron Nozzle Mechanisms and Trends, AIAA Journal 43 (2005) 87-95.

[26] M. B. Alkislar, A. Krothapalli, G. W. Butler, The effect of streamwise vortices on the aeroacoustics of a Mach 0.9 jet, Journal of Fluid Mechanics 578 (2007) 139.

[27] P. Tide, K. Srinivasan, Effect of chevron count and penetration on the acoustic characteristics of chevron nozzles, Applied Acoustics 71 (2010) 201-220.

[28] S. R. Nikam, S. D. Sharma, Effect of chevron nozzle penetration on aero-acoustic characteristics of jet at M $=0.8$, Fluid Dynamics Research 49 (2017) 065506.

[29] A. Uzun, M. Y. Hussaini, Simulation of Noise Generation in the Near-Nozzle Region of a Chevron Nozzle Jet, AIAA Journal 47 (2009) 1793-1810.

[30] A. Uzun, M. Y. Hussaini, Some Issues in Large-Eddy Simulations for Chevron Nozzle Jet Flows, Journal of Propulsion and Power 28 (2012) 246-258.

[31] H. Xia, P. G. Tucker, Numerical Simulation of Single-Stream Jets from a Serrated Nozzle, Flow, Turbulence and Combustion 88 (2012) 3-18

[32] H. Xia, Turbulent jet characteristics for axisymmetric and serrated nozzles, Computers \& Fluids 110 (2015) $189-197$.

[33] M. O. Cetin, V. Pauz, M. Meinke, W. Schröder, Computational analysis of nozzle geometry variations for subsonic turbulent jets, Computers \& Fluids 136 (2016) 467-484.

[34] Z.-N. Wang, J. C. Tyacke, P. G. Tucker, LES-RANS of installed ultra-high bypass-ratio coaxial jet aeroacoustics with a finite span wing-flap geometry and flight stream - Part 2: chevron nozzle, AIAA 2017-3855.

[35] C. Bailly, C. Bogey, Contributions of Computational Aeroacoustics to Jet Noise Research and Prediction, International Journal of Computational Fluid Dynamics 18 (2004) 481-491. 
[36] N. N. Pastouchenko, C. K. Tam, Installation Effects on the Flow and Noise of Wing Mounted Jets, AIAA Journal 45 (2007) 2851-2860.

[37] U. Paliath, S. Premasuthan, Large Eddy Simulation for Jet Installation Effects, AIAA 2013-2137.

[38] M. Angelino, H. Xia, G. J. Page, Adaptive Wall-Modelled Large Eddy Simulation of Jet Noise in Isolated and Installed Configurations, American Institute of Aeronautics and Astronautics, 2018.

[39] M. Angelino, M. A. Moratilla-Vega, A. Howlett, H. Xia, G. J. Page, Numerical Investigation of Installed Jet Noise Sensitivity to Lift and Wing/Engine Positioning, in: 25th AIAA/CEAS Aeroacoustics Conference, American Institute of Aeronautics and Astronautics, Delft, The Netherlands, 2019.

[40] C. Bogey, O. Marsden, A study of the grid dependence of the flow field and noise of subsonic jets, AIAA 2016-0261.

[41] H. Tanna, An experimental study of jet noise part II: Shock associated noise, Journal of Sound and Vibration 50 (1977) $429-444$.

[42] B. van Leer, Towards the ultimate conservative difference scheme. V. A second-order sequel to Godunov's method, Journal of Computational Physics 32 (1979) 101-136.

[43] A. Jameson, Time dependent calculations using multigrid, with applications to unsteady flows past airfoils and wings, in: 10th Computational Fluid Dynamics Conference, American Institute of Aeronautics and Astronautics, Honolulu,HI,U.S.A., 1991.

[44] P. Roe, Approximate Riemann solvers, parameter vectors, and difference schemes, Journal of Computational Physics 43 (1981) 357-372.

[45] M.-S. Liou, C. J. Steffen, A New Flux Splitting Scheme, Journal of Computational Physics 107 (1993) $23-39$.

[46] E. Shima, K. Kitamura, Parameter-Free Simple Low-Dissipation AUSM-Family Scheme for All Speeds, AIAA Journal 49 (2011) 1693-1709.

[47] A. Jameson, Formulation of Kinetic Energy Preserving Conservative Schemes for Gas Dynamics and Direct Numerical Simulation of One-Dimensional Viscous Compressible Flow in a Shock Tube Using Entropy and Kinetic Energy Preserving Schemes, Journal of Scientific Computing 34 (2008) 188-208.

[48] T. T. Bui, A parallel, finite-volume algorithm for large-eddy simulation of turbulent flows, Computers \& Fluids 29 (2000) $877-915$.

[49] H. Xia, Dynamic Grid Detach-Eddy Simulation for Synthetic Jet Flows, PhD thesis, University of Sheffield, 2005.

[50] A. Boghi, M. Angelino, F. Gori, Numerical evidence of an undisturbed region of flow in a turbulent rectangular submerged free jet, Numerical Heat Transfer, Part A: Applications 70 (2016) 14-29.

[51] M. Angelino, A. Boghi, F. Gori, Numerical solution of three-dimensional rectangular submerged jets with the evidence of the undisturbed region of flow, Numerical Heat Transfer, Part A: Applications 70 (2016) 815-830.

[52] I. Di Venuta, I. Petracci, M. Angelino, A. Boghi, F. Gori, Numerical simulation of mass transfer and fluid flow evolution of a rectangular free jet of air, International Journal of Heat and Mass Transfer 117 (2018) 235-251.

[53] I. Di Venuta, A. Boghi, M. Angelino, F. Gori, Passive scalar diffusion in three-dimensional turbulent rectangular free jets with numerical evaluation of turbulent Prandtl/Schmidt number, International Communications in Heat and Mass Transfer 95 (2018) 106-115.

[54] F. F. Grinstein, C. Fureby, Recent Progress on MILES for High Reynolds Number Flows, Journal of Fluids Engineering $124(2002) 848$.

[55] P. G. Tucker, Novel MILES computations for jet flows and noise, International Journal of Heat and Fluid Flow 25 (2004) 
$625-635$.

[56] F. Nicoud, F. Ducros, Subgrid-Scale Stress Modelling Based on the Square of the Velocity Gradient Tensor, Flow, Turbulence and Combustion 62 (1999) 183-200.

[57] A. W. Vreman, An eddy-viscosity subgrid-scale model for turbulent shear flow: Algebraic theory and applications, Physics of Fluids 16 (2004) 3670-3681.

[58] F. Nicoud, H. B. Toda, O. Cabrit, S. Bose, J. Lee, Using singular values to build a subgrid-scale model for large eddy simulations, Physics of Fluids 23 (2011) 085106.

[59] M. Angelino, M. A. Moratilla-Vega, H. Xia, G. J. Page, Large-eddy simulations of high Reynolds number jets with a suitable subgrid-scale model for solver dependency study, in: 11th International ERCOFTAC Symposium on Engineering Turbulence Modelling and Measurements, Palermo, Italy.

[60] C. Bogey, O. Marsden, C. Bailly, Influence of initial turbulence level on the flow and sound fields of a subsonic jet at a diameter-based Reynolds number of 105, Journal of Fluid Mechanics 701 (2012) 352-385.

[61] C. Bogey, O. Marsden, Identification of the effects of the nozzle-exit boundary-layer thickness and its corresponding Reynolds number in initially highly disturbed subsonic jets, Physics of Fluids 25 (2013) 055106.

[62] A. Uzun, M. Y. Hussaini, Prediction of Noise Generated by a Round Nozzle Jet Flow Using Computational Aeroacoustics, Journal of Computational Acoustics 19 (2011) 291-316.

[63] P. Di Francescantonio, A new boundary integral formulation for the prediction of sound radiation, Journal of Sound and Vibration 202 (1997) 491-509.

[64] M. Shur, P. Spalart, M. Strelets, A. Travin, Towards the prediction of noise from jet engines, International Journal of Heat and Fluid Flow 24 (2003) 551-561.

[65] J. C. R. Hunt, A. A. Wray, P. Moin, Eddies, Streams, and Convergence Zones in Turbulent Flows, in: Proceedings of the Summer Program, N89-24555, NASA Center for Turbulence Research.

[66] T. Suzuki, T. Colonius, Instability waves in a subsonic round jet detected using a near-field phased microphone array, Journal of Fluid Mechanics 565 (2006) 197.

[67] J. Bridges, M. Wernet, Establishing Consensus Turbulence Statistics for Hot Subsonic Jets, AIAA $2010-3751$.

[68] G. I. Taylor, The Spectrum of Turbulence, Proceedings of the Royal Society A: Mathematical, Physical and Engineering Sciences 164 (1938) 476-490.

[69] S. B. Pope, Turbulent flows, Cambridge University Press, 2000.

[70] M. Gloor, S. Bühler, L. Kleiser, Transition to turbulence and noise radiation in heated coaxial jet flows, Physics of Fluids 28 (2016) 044103.

[71] C. Brown, J. Bridges, Small Hot Jet Acoustic Rig Validation, NASA Technical Memorandum $214234,2006$.

[72] A. V. Cavalieri, G. Daviller, P. Comte, P. Jordan, G. Tadmor, Y. Gervais, Using large eddy simulation to explore sound-source mechanisms in jets, Journal of Sound and Vibration 330 (2011) 4098-4113.

[73] M. Lorteau, F. Cléro, F. Vuillot, Analysis of noise radiation mechanisms in hot subsonic jet from a validated large eddy simulation solution, Physics of Fluids 27 (2015) 075108.

[74] G. A. Brès, V. Jaunet, M. Le Rallic, P. Jordan, A. Towne, O. Schmidt, T. Colonius, A. V. Cavalieri, S. K. Lele, Large eddy simulation for jet noise: azimuthal decomposition and intermittency of the radiated sound, AIAA 2016-3050.

[75] A. V. G. Cavalieri, P. Jordan, T. Colonius, Y. Gervais, Axisymmetric superdirectivity in subsonic jets, Journal of Fluid Mechanics 704 (2012) 388-420. 
[76] G. Bodard, C. Bailly, F. Vuillot, Matched Hybrid Approaches to Predict Jet Noise by Using Large Eddy Simulation, AIAA 2009-3316.

[77] C. K. W. Tam, L. Auriault, Jet Mixing Noise from Fine-Scale Turbulence, AIAA Journal 37 (1999) $145-153$.

[78] M. Vinokur, On one-dimensional stretching functions for finite-difference calculations, Journal of Computational Physics 50 (1983) 215-234. 